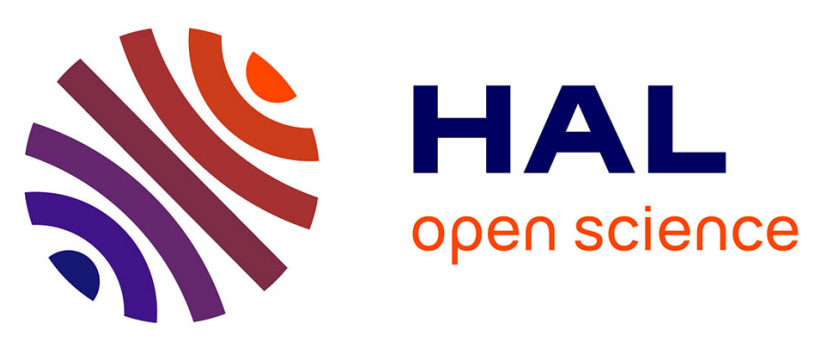

\title{
3D coupled HM-XFEM modeling with cohesive zone model and applications to non planar hydraulic fracture propagation and multiple hydraulic fractures interference
}

Bertrand Paul, Maxime Faivre, P. Massin, Richard Giot, Daniele Colombo, F. Golfier, Alexandre Martin

\section{To cite this version:}

Bertrand Paul, Maxime Faivre, P. Massin, Richard Giot, Daniele Colombo, et al.. 3D coupled HMXFEM modeling with cohesive zone model and applications to non planar hydraulic fracture propagation and multiple hydraulic fractures interference. Computer Methods in Applied Mechanics and Engineering, 2018, 342, pp.321 - 353. 10.1016/j.cma.2018.08.009 . hal-01881975v2

\author{
HAL Id: hal-01881975 \\ https://hal.science/hal-01881975v2
}

Submitted on 20 Sep 2018

HAL is a multi-disciplinary open access archive for the deposit and dissemination of scientific research documents, whether they are published or not. The documents may come from teaching and research institutions in France or abroad, or from public or private research centers.
L'archive ouverte pluridisciplinaire HAL, est destinée au dépôt et à la diffusion de documents scientifiques de niveau recherche, publiés ou non, émanant des établissements d'enseignement et de recherche français ou étrangers, des laboratoires publics ou privés. 


\title{
3D coupled HM-XFEM modeling with cohesive zone model and applications to non planar hydraulic fracture propagation and multiple hydraulic fractures interference
}

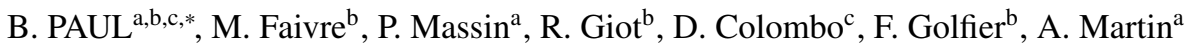 \\ ${ }^{a}$ IMSIA, UMR EDF-CNRS-CEA-ENSTA ParisTech, Universit Paris-Saclay, 7 boulevard Gaspard Monge, 91120 Palaiseau, France \\ ${ }^{b}$ Université de Lorraine, ENSG; CNRS ; CREGU; GeoRessources Laboratory, F-54501 Vandoeuvre-Lès-Nancy, France. \\ ${ }^{c}$ IFP Energies Nouvelles, 92852 Rueil-Malmaison Cedex, France
}

\begin{abstract}
A 3D fully coupled hydromechanical model for the simulation of fluid-driven fracture propagation through poroelastic saturated media is presented and compared to several analytical or numerical benchmarks. The hydromechanical coupling in the porous matrix is derived within the framework of the generalized Biot theory and the fluid flow in the fractures satisfies the lubrication equation. The presence and propagation of fluid-driven fractures is handled with the extended finite element method and the propagation of the fluid-driven fractures is governed by a mixed linear cohesive law relying on a stable mortar formalism. A comparison between numerical results and a semi-analytical solution for plane fluid-driven fractures in porous media assess the validity of the proposed model. Then, a procedure for the propagation of fluid-driven fractures on non predefined paths is detailed. In particular, the fracture reorientation angle is computed exclusively from cohesive quantities. Various numerical experiments are performed to study the interferences between neighboring fluid-driven fractures as well as the reorientation of fluid-driven fractures under complex stress conditions. Finally, the model is extended to discontinuity junctions and an application to arrays of vertical fractures initiated from horizontal wells is presented.
\end{abstract}

Keywords: X-FEM, hydro-mechanical coupling, fluid-driven fracture, cohesive zone model, fracture spacing, fracture interference, complex fracture

\section{Introduction}

Predicting occurrence of hydraulically induced damage in geological systems constitutes a major challenge in subsurface engineering. Geo-resource completion (geothermal, oil and gas resources), underground storage management (confinement of hazardous wastes, $\mathrm{CO}_{2}$ storage) or building and maintenance of constructions (tunnels, dams, mines) can be affected by the progressive development of cracks due to stress relaxation and fluid flow circulation inside the host formation. Hydro-mechanical (HM) processes may be caused by natural or anthropic forcing that need to be understood and modeled for a variety of problems. Large injections of gas $\left(\mathrm{CO}_{2}, \mathrm{H}_{2}\right)$ in underground reservoir storages can result in significant pressure build-up which can affect the stress field and induce large deformations or initiate induced seismicity [1]. These HM processes can eventually damage the cap rock and open up new flow paths which, ultimately, could ruin the efforts made for keeping the gas stored underground. There is, therefore, a critical need to build capable numerical models (fully coupled, multi-scale, etc) to gain better insights into these complex phenomena and thus to improve their predictive capabilities.

During the last decades, the problem of fluid-driven fracture propagation has been tackled analytically ([2], [3], [4]) and more recently by different numerical approaches $([5],[6])$. The analytical effort on this subject aimed at solving

*. Corresponding author at : IMSIA, UMR EDF-CNRS-CEA-ENSTA ParisTech, Universit Paris-Saclay, 7 boulevard Gaspard Monge, 91120 Palaiseau, France

Email address: bertrand.paul@polytechnique.org (B. PAUL) 


\begin{tabular}{|c|c|}
\hline \multicolumn{2}{|c|}{ Nomenclature } \\
\hline$b$ & Biot's coefficient \\
\hline$C_{L}$ & leak-off coefficient \\
\hline$C$ & Hooke's elasticity tensor \\
\hline E & Young's modulus \\
\hline$E^{\prime}$ & plane strain modulus \\
\hline$G_{c}$ & cohesive energy \\
\hline$K_{c}$ & fracture toughness for mode I \\
\hline$k^{\text {int }}$ & intrinsic permeability \\
\hline$K_{s}$ & bulk compressibility modulus \\
\hline$K_{w}$ & fluid compressibility modulus \\
\hline$l s n$ & normal level-set \\
\hline lst & tangential level-set \\
\hline $\mathscr{L}$ & augmented Lagrangian \\
\hline $\mathscr{M}_{k}$ & dimensionless viscosity \\
\hline$M$ & mass flux in the bulk \\
\hline$n$ & outward normal to the fracture \\
\hline$\Pi$ & surface density of energy \\
\hline$p$ & pore pressure field in the porous medium \\
\hline$p_{f}$ & fluid pressure field inside the cohesive fracture \\
\hline$q_{-}, q_{+}$ & Lagrange multipliers (hydrodymamical part) \\
\hline$r$ & normal augmentation parameter \\
\hline$r_{s}$ & tangential augmentation parameter \\
\hline$r^{\text {hom }}$ & homogenized density of the porous medium \\
\hline$t$ & time \\
\hline$t^{*}$ & characteristic time for the penny shape problem \\
\hline$t_{c}$ & total cohesive stress \\
\hline$t_{c_{n}}$ & normal total cohesive stress \\
\hline$t_{c}^{\prime}$ & effective cohesive stress \\
\hline$t_{c_{n}}^{\prime c}$ & normal effective cohesive stress \\
\hline$T_{d}$ & damage tensor \\
\hline$u$ & nodal displacement field \\
\hline$\llbracket u \rrbracket$ & nodal displacement jump \\
\hline $\boldsymbol{W}$ & mass flux in the cohesive fracture \\
\hline$w$ & displacement jump \\
\hline$w_{c}$ & critical opening \\
\hline$w_{n}$ & normal displacement jump \\
\hline$\alpha$ & internal variable for the cohesive law \\
\hline$\beta$ & reorientation angle \\
\hline$\lambda, \mu$ & Lagrange multipliers (mechanical part) \\
\hline$\mu$ & dynamic viscosity \\
\hline$v$ & Poisson's ratio \\
\hline$\rho$ & fluid density \\
\hline$\sigma_{c}$ & critical stress \\
\hline$\sigma_{0}$ & confining stress \\
\hline$\phi$ & eulerian porosity \\
\hline
\end{tabular}

the complex system of non linear partial derivative equations governing these phenomena. Asymptotic solutions were 
derived such as the Kristianovic-Geertsma-de Klerk (KGD) or Perkins-Kern-Nordgren (PKN) models but they are restricted to simple configurations involving plane fracture paths [7], [8], [9].

In parallel, various numerical approaches have been used to investigate hydraulic fracture propagation. For example, damage models have been adapted to saturated geo-materials [10], [11]. Damage internal variables are updated locally depending on the pore pressure and strain state, rendering the evolution of micro-cracks that will engender a macro-crack by coalescence. Nevertheless, these models do not account for the plastic deformations that are essential to describe the damage process. To tackle this issue, some authors proposed micro-mechanical damage models [12], able to model the micro-structure of brittle materials. Nonetheless, these micro-mechanical models are not adequate for the modeling of fracture whose aperture may not be neglected anymore.

Henceforth when the number of fractures to simulate is small, we can consider to model explicitly the fractures kinematics using the finite element method, for instance with interface elements [6], [13], [14]. It is then possible to account for the preferential fluid flow in the fractures but it requires computationally expensive remeshing techniques to deal with fracture growth. In that regard, most of the models use the cubic law to model the flow along fracture paths, as justified in [15] even if in the crack tip region, the fluid lag effect is a matter of great concern [16]. The strong non linear hydromechanical coupling, indeed, can lead to a singularity of the fluid pressure due to the delay of the fluid front compared to the crack front advance. The unsaturated cavity that appears ahead of the fluid front is then either filled with fluid vapor or with pore-fluid infiltrating from the bulk [17]. Other numerical methods considering discrete fractures include the discrete element method [18], the HM lattice approach [19] and the discrete fracture network [20].

In order to circumvent the difficulty of generating a mesh conforming the fracture network, some authors have coupled hydromechanical models with the eXtended Finite Element Method (XFEM). The XFEM has been introduced by Belytschko et al. [21] and Moës et al. [22]. It is based on the partition of the unity [23]. It allows to handle discontinuities within the mesh via additional degrees of freedom associated to discontinuous shape functions. The combination of hydromechanical models with the XFEM seems effectively appropriate for the simulation of fluiddriven fractures propagation, in particular for complex fracture geometries. Indeed, when fractures are discretized with interface elements, it is necessary to generate a mesh that matches fractures geometries, which can be very difficult for 3D-non planar connected fractures. In addition, it requires to update the mesh at each propagation step, involving projection algorithms that are expensive. The XFEM circumvents these difficulties.

Most of the hydromechanical models coupled with XFEM found in the literature consider CZM (Cohesive Zone Model) based models [24] to handle the crack tip behavior. When the fluid pressure reaches a threshold inherent to the CZM, the damage process starts and the fracture propagates, releasing an empty space for the fluid. Alternative approaches like the gradient approaches [25], the phase-field models [26, 27], [28] or non local approaches relying on regularization techniques [29], [30] assume a diffuse deterioration of the bulk. The use of a cohesive zone model is very practical as it authorizes the advancement of the crack front on a predefined path within a time step, as the mechanical equilibrium is established. An alternative consists in using a singular enrichment at the tip as suggested by Lecampion [31]. Depending on the propagation regime (viscosity dominated or toughness dominated), the singular enrichment is adapted to fit with the correct asymptotic behavior at the tip. Gordeliy and Peirce [32] proved that this approach allows to recover optimal convergence rates for the analytical asymptotic solutions detailed in [33].

XFEM models have been considered for single fluid flow [34], [35], [36], [37], [38] or two fluid flow [39], [40]. The influence of the thermal effects has also been studied [41], [35]. Besides the petroleum engineering applications [36], [37], these models are employed to analyze the harmfulness of cracks in civil engineering buildings [42]. For example, a specific model for the propagation of fluid-driven fractures in concrete has been developed by Ren et al. [43]. During the last years, XFEM models became more and more sophisticated. Huygue and Remij [44] have simulated the reorientation of fractures depending on the regional stress state. We can also cite the model developed by Bunger and Peirce [45] that is able to handle several fractures in the vicinity of a well in order to appreciate the competition that takes place between nearby fractures. Interaction and junction between multiple fractures have been studied by Khoei et al. [46] and Shi et al. [47]. Recently, an XFEM-based cohesive zone model combined with Mohr-Coulomb theory of plasticity was also developed to investigate non-planar fracture propagation in both brittle and ductile formations [48, 49]. Reorientation of "wing cracks" [48] and interferences between nearby fluid-driven fractures [49] for such materials were more specifically considered. Finally, extension to 3D fracture geometries was initiated by Gupta and Duarte [50] with an adaptative GFEM model and Haddad and Spehrnoori [51] using the XFEM-based CZM developed in the commercial software Abaqus but for high computational restrictions due to 
dynamic mesh refinement.

Up to now, however, all of these XFEM models suffer several drawbacks. First, few of these models consider a fully coupled approach and fluid flow in the fractures is often described in a simplified way using a 1D leak-off model for instance [52]. Réthoré et al. proposed such an uncoupled model [53] where the fluid flow in the fracture is simulated independently from the rest of the problem. It was later extended to the partially saturated case [54]. This two-scale approach enables a fine simulation of the fluid flow in the fractures but it fails in rendering the hydrodynamical coupling between the fluid filled fracture and the porous medium. Alternatively, some models [55] insert interface elements in the XFEM model in order to simulate leak off. The fluid loss from the interface to the porous matrix is then proportional to the pressure gap between the interface and the porous matrix but this is only acceptable for fractures whose geometry is simple and that do not interact with nearby fractures. Second, the vast majority of XFEM models are limited to short fractures propagation where plane strain assumptions are valid and are very far to be able to describe a 3D realistic configuration with complex fracture geometries. Moreover, if a certain number of numerical challenges (e.g., non planar fracture, 3D configuration, fracture junction, plasticity) have been identified, they have been tackled in a dispersed way as mentioned above and all of the models fail to address them simultaneously.

The aim of this study is to develop a 3D numerical model of hydraulic fracture propagation that can overcome the challenges discussed above. This XFEM model is based on the previous numerical model developped by Faivre et al. [34] but with the following distinguished features : (i) fully hydromechanical coupling based on an improved XFEM enrichment technique, (ii) fracture propagation on non-predefined paths, (iii) 3D configuration including complex nonplanar fracture geometries and (iv) multiple-crack junction. Besides the extension of our model to fluid-filled cohesive crack junctions, most of these new features borrow on recently published advances on the XFEM [56, 57, 58, 59]. The assembly of all these state-of-the art techniques results in a more complete model which is a step forward to address the challenges mentioned above.

In the present work, the hydromechanical coupling is fully satisfied because the interstitial fluid and the fluid in the fractures form a single fluid phase through the continuity conditions imposed at the fracture walls. This is a significant advantage compared to partially coupled approaches, in particular to assess the competition between the leak-off phenomenon and the sustaining of the fractures under the action of the pressurized fluid for complex fracture geometries. For the propagation of cohesive hydraulic fractures on non-predefined paths, we rely on the approach developed by Ferté [58] for brittle failure. The bifurcation angle is determined by means of the stress intensity factors computed with cohesive integrals only. Alternative techniques rely on local criteria for the determination of the bifurcation angle. In order to overcome conditioning issues usually encountered with 3D XFEM elements, a robust XFEM enrichment technique is employed [56]. It is associated to a consistent integration technique adapted to curved cracks and branched discontinuities [57]. The stability of our model is ensured by the use of three distinct approximation spaces. The displacements field is interpolated in a quadratic way, the pore pressure field is interpolated in a linear way and finally, fields associated to the cohesive fractures are discretized over an appropriate reduced approximation space based on vertex nodes of edges intersected by the fracture. That way, we do not observe spurious oscillations in the numerical resolution. Note that the HM-XFEM model presented in the paper has been implemented in Code_Aster (http://web-code-aster.org).

In the following, we introduce first the theoretical framework of this hydromechanical model coupled with XFEM and able to model the propagation of fluid-driven fractures on predefined paths thanks to a cohesive zone model. In particular, we extend our model to 3D geometries and introduce a new "mortar" formulation for the cohesive zone model, inspired by the work of Ferté [58]. The next section is dedicated to the validation of the model on the pennyshaped benchmark. Then the mathematical procedure for the propagation of fluid-driven fractures on non-predefined paths, based on the work of Ferté [58] is discussed. Several examples of fracture reorientation and competition between nearby fractures are proposed. Finally, we extend our model to branched hydraulic fractures. A preliminary example illustrates the range of potential applications allowed by this additional functionality.

\section{Overview of the mortar HM-XFEM model}

\subsection{Hypothesis and notations}

We consider a cohesive fracture $\Gamma_{c}$ included in a bounded domain $\Omega$. The domain $\Omega$ is saturated by a single fluid phase characterized by a pore pressure $p$. The fluid pressure in the cohesive fracture is denoted $p_{f}$. The mass 
exchanges from the fracture to the porous medium at the upper fracture wall $\Gamma_{+}$and lower fracture wall $\Gamma_{-}$are denoted respectively $q_{+}$and $q_{-}$(see Figure 1 . Along the domain boundary $\partial \Omega$, we impose :

- the displacements $\boldsymbol{u}$ to zero on $\Gamma_{u}$,

- the pore pressure $p$ to zero on $\Gamma_{p}$,

- a stress $t$ on surface $\Gamma_{t}$,

- flux $M_{\text {ext }}$ on surface $\Gamma_{F}$,

- flux $W_{\text {ext }}$ at the cohesive fracture inlet $\Gamma_{f}$ (refers to the borehole shrunk to a point).

We denote $r^{\text {hom }}$ the homogenized density of the porous matrix.

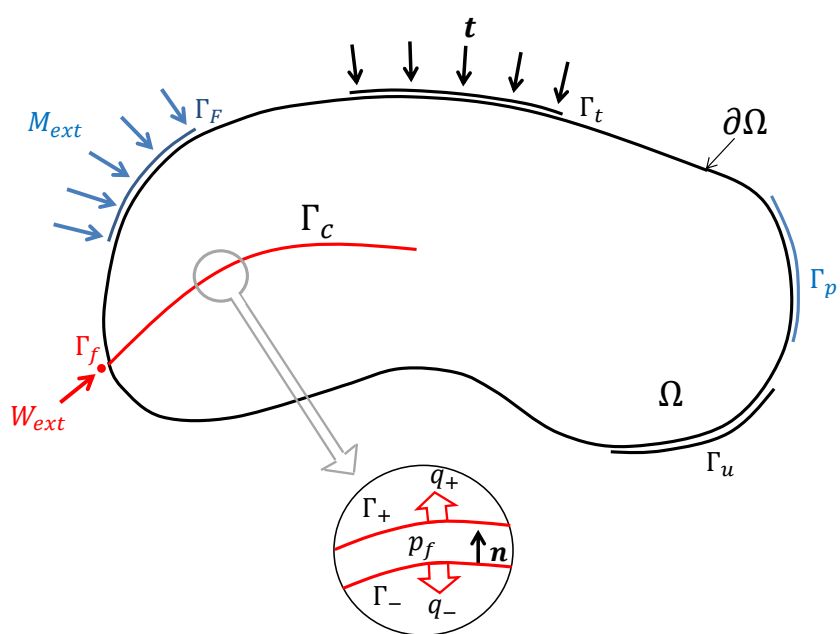

FIGURE 1: Domain decomposition and loadings.

The displacement jump is defined as follows :

$$
\llbracket \boldsymbol{u} \rrbracket \cdot \boldsymbol{n}=\left[\boldsymbol{u}\left(\Gamma_{+}\right)-\boldsymbol{u}\left(\Gamma_{-}\right)\right] \cdot \boldsymbol{n} \geq 0
$$

In addition, infinitesimal strain and Biot effective stress are assumed.

\subsection{Governing equations of the hydrodynamical problem}

In this paragraph, we depict the set of equations governing the fluid phase. In particular, the homogeneity between the interstitial fluid and the fluid in the cohesive fracture is ensured by continuity conditions enforced at the fracture walls.

\subsubsection{Mass balance for the fluid in the bulk}

The interstitial fluid, with pore pressure $p$ is governed by the mass balance equation :

$$
\frac{\partial\left(\rho \phi\left(1+\varepsilon_{v}\right)\right)}{\partial t}+\operatorname{Div}(\boldsymbol{M})=0
$$

where $\rho$ is the fluid density, $\phi$ the eulerian porosity of the bulk, $\varepsilon_{v}=\operatorname{Tr}(\boldsymbol{\nabla u})=\operatorname{Tr}(\boldsymbol{\varepsilon})$ the volumetric strain and $\boldsymbol{M}$ the fluid flux.

The variations of the fluid density and of the porosity are respectively of the form :

$$
\frac{d \rho}{\rho}=\frac{d p}{K_{w}}
$$


and

$$
d \phi=(b-\phi)\left(d \varepsilon_{v}+\frac{d p}{K_{s}}\right)
$$

where $K_{s}$ is the bulk compressibility modulus for the solid phase, $K_{w}$ the fluid compressibility modulus and $b$ Biot's coefficient [60].

Finally, the mass flux for a fully-saturated porous medium is given by Darcy's law (gravity is neglected) :

$$
\boldsymbol{M}=-\rho \frac{k^{i n t}}{\mu} \nabla p
$$

where $k^{\text {int }}$ represents the intrinsic permeability and $\mu$ the dynamic viscosity.

\subsubsection{Mass balance for the fluid in the fracture}

In the cohesive fracture, the fluid pressure is denoted $p_{f}$ and source terms $q_{+}$and $q_{-}$may occur from the fracture to the surrounding porous medium so that the mass balance equation is :

$$
\frac{\partial(\rho \llbracket \boldsymbol{u} \rrbracket \cdot \boldsymbol{n})}{\partial t}+\operatorname{Div}(\boldsymbol{W})=q_{-}+q_{+}
$$

where $\boldsymbol{W}$ is the fluid flux inside the fracture. Once again, we assume that the fluid flux depends on the gradient of the fluid pressure, but that the conductivity depends on the cube of the aperture so that the fluid flow $\boldsymbol{W}$ is given by the cubic law [15] (still neglecting the gravity effects) :

$$
\boldsymbol{W}=-\frac{\rho(\llbracket \boldsymbol{u} \rrbracket \cdot \boldsymbol{n})^{3}}{12 \mu} \nabla p_{f}
$$

Finally, the variation of the fluid density inside the fracture is given by :

$$
\frac{d \rho}{\rho}=\frac{d p_{f}}{K_{w}}
$$

\subsubsection{Fluid pressure continuity at the fracture walls}

In order to ensure the continuity of the fluid pressure and the pore pressure, we impose the relation $p=p_{f}$ at both fracture walls :

- $p=p_{f}$ on $\Gamma_{-}$

- $p=p_{f}$ on $\Gamma_{+}$

As mentioned in the introduction, the pore pressure field is enriched with XFEM so that it is eventually discontinuous across the fracture surface. We impose the continuity of the fluid pressure at both fracture walls to make sure that only the gradient of the pore pressure field is discontinuous. These conditions are weakly enforced with the source terms $q_{-}$and $q_{+}$, interpreted as Lagrange multipliers.

\subsubsection{Weak formulation of the hydrodynamical problem}

The weak form of the hydrodynamical problem is composed of 4 equations : the two mass balance equations and the two equations for the fluid pressure continuity along each fracture side. Both mass balance equations are discretized in time with a $\theta$-scheme that is unconditionally stable for $\theta \geq \frac{1}{2}$ and of order 1 except when $\theta=\frac{1}{2}$ for which it is of order 2. A value of $\theta \neq \frac{1}{2}$ will be chosen in order to avoid spurious oscillations. The superscript + indicates that the variable is expressed at the current time-step whereas the superscript - refers to a variable expressed at the previous time-step, and $\Delta t=t^{+}-t^{-}$. 
According to the principle of Virtual Work, the weak formulation of the mass balance equation for the interstitial fluid is :

$$
\begin{aligned}
\forall p^{*} \in P_{0} \quad & -\int_{\Omega} \frac{m_{w}^{+}-m_{w}^{-}}{\Delta t} p^{*} d \Omega+\theta \int_{\Omega} \boldsymbol{M}^{+} \cdot \nabla p^{*} d \Omega+(1-\theta) \int_{\Omega} \boldsymbol{M}^{-} \cdot \nabla p^{*} d \Omega \\
& =\theta \int_{\Gamma_{F}} M_{e x t}^{+} p^{*} d \Gamma_{F}+(1-\theta) \int_{\Gamma_{F}} M_{e x t}^{-} p^{*} d \Gamma_{F}-\theta \int_{\Gamma_{-}} q_{-}^{+} p^{*} d \Gamma_{-} \\
& -(1-\theta) \int_{\Gamma_{-}} q_{-}^{-} p^{*} d \Gamma_{-}-\theta \int_{\Gamma_{+}} q_{+}^{+} p^{*} d \Gamma_{+}-(1-\theta) \int_{\Gamma_{+}} q_{+}^{-} p^{*} d \Gamma_{+}
\end{aligned}
$$

with : $P_{0}=\left\{p^{*} \in H^{1}(\Omega)\right.$ such that $p^{*}$ discontinous through $\Gamma_{c}$ and $p^{*}=0$ on $\left.\Gamma_{p}\right\}$ and $m_{w}^{+}-m_{w}^{-}=\rho^{+} \phi^{+}\left(1+\varepsilon_{v}^{+}\right)-$ $\rho^{-} \phi^{-}\left(1+\varepsilon_{v}^{-}\right)$.

In the same manner, the weak formulation of the mass balance equation for the fluid in the cohesive fracture is :

$$
\begin{aligned}
\forall p_{f}^{*} \in M_{0} & -\int_{\Gamma_{c}} \frac{w^{+}-w^{-}}{\Delta t} p_{f}^{*} d \Gamma_{c}+\theta \int_{\Gamma_{c}} \boldsymbol{W}^{+} \cdot \nabla p_{f}^{*} d \Gamma_{c}+(1-\theta) \int_{\Gamma_{c}} \boldsymbol{W}^{-} \cdot \nabla p_{f}^{*} d \Gamma_{c} \\
& =\theta \int_{\Gamma_{f}} W_{e x t}^{+} p_{f}^{*} d \Gamma_{f}+(1-\theta) \int_{\Gamma_{f}} W_{e x t}^{-} p_{f}^{*} d \Gamma_{f}+\theta \int_{\Gamma_{-}} q_{-}^{+} p_{f}^{*} d \Gamma_{-} \\
& +(1-\theta) \int_{\Gamma_{-}} q_{-}^{-} p_{f}^{*} d \Gamma_{-}+\theta \int_{\Gamma_{+}} q_{+}^{+} p_{f}^{*} d \Gamma_{+}+(1-\theta) \int_{\Gamma_{+}} q_{+}^{-} p_{f}^{*} d \Gamma_{+}
\end{aligned}
$$

with : $M_{0}=\left\{\Lambda^{*} \in H^{-\frac{1}{2}}\left(\Gamma_{c}\right)\right\}$ and $w^{+}-w^{-}=\rho^{+} \llbracket \boldsymbol{u} \rrbracket^{+} \cdot \boldsymbol{n}_{c}-\rho^{-} \llbracket \boldsymbol{u} \rrbracket^{-} \cdot \boldsymbol{n}_{c}$.

To finish, the weak formulation of the pressure continuity along both fracture walls is given by :

$$
\begin{array}{ll}
\forall q_{-}^{*} \in M_{0} & \int_{\Gamma_{-}}\left(p-p_{f}\right) q_{-}^{*} d \Gamma_{-}=0 \\
\forall q_{+}^{*} \in M_{0} \quad \int_{\Gamma_{+}}\left(p-p_{f}\right) q_{+}^{*} d \Gamma_{+}=0
\end{array}
$$

\subsection{Mortar formulation for the cohesive zone model}

In this paragraph, we present a new "mortar" formulation for the cohesive zone model, which differs from what was exposed in [34]. Instead of inserting the cohesive zone model by collocation at each Gauss point based on the augmented Lagrangian formalism of Lorentz [61], we rely on the "mortar" formalism introduced by Ferté [58]. With the formulation of Ferté, the whole internal variables set relative to the cohesive fracture is carried by the vertex nodes of the edges intersected by the discontinuity. In this way, we are able to implicitly locate the position of the cohesive crack front with a set of nodal values, compatible from one element to the other. This feature will be crucial in the procedure for the propagation of fluid-driven cohesive fractures on non predefined paths (section 4). Additionally, the Talon-Curnier cohesive law we previously employed [34] is replaced by a mixed linear cohesive law. This mixed linear cohesive law enhances the stability of our numerical model as it behaves elastically for unloading situations instead of traction free unloading for the Talon-Curnier cohesive law.

\subsubsection{Mixed linear non-regularized cohesive law}

As mentioned in the introduction, we use a cohesive zone model along the fluid-driven fracture path (see Figure 2 left). The fracture is then decomposed into three distinct zones :

- an entirely opened zone (zone 1) where the total stress on both fracture walls is equal to $-p_{f} \boldsymbol{n}$ with $\boldsymbol{n}$ a unit vector, normal to the fracture walls, pointing from the porous matrix to the cohesive fracture ;

- a partially opened zone (zone 2) where the total stress on the fracture walls (under the assumption of the effective stress) is $\boldsymbol{t}_{\boldsymbol{c}}=\boldsymbol{t}_{\boldsymbol{c}}^{\prime}-p_{f} \boldsymbol{n}$ with $\boldsymbol{t}_{\boldsymbol{c}}^{\prime}$ the effective cohesive stress ; 
- an undamaged zone (zone 3) where the two sides of the discontinuity are in perfect adhesion and no interpenetration occurs.

The damage process takes place in zone 2 in which the effective cohesive stress is directly linked to the aperture via a linear softening relation (see Figure 2 right). Over a critical fracture opening $w_{c}$ the effective cohesive stress vanish and the total stress is equal to the stress defined in zone 1 . At the limit between the partially opened zone and the undamaged zone, the effective cohesive stress reaches the critical stress $\sigma_{c}$. It is the location of the cohesive crack front.

Let us call $\boldsymbol{w}$ the displacement jump across the cohesive fracture. Following the notations of Ferté [58], an expression for the surface density of energy in the augmented Lagrangian formalism is :

$$
\Pi(\boldsymbol{w}, \lambda)=\psi\left(\lambda_{n}+r w_{n}, \lambda_{s}+r_{s} \boldsymbol{w}_{s}\right)-\frac{\lambda_{n}^{2}}{2 r}-\frac{\lambda_{s} \cdot \lambda_{s}}{2 r_{s}}
$$

with $\psi$ a differentiable function depending on the shape of the cohesive law, $r$ and $r_{s}$ the normal and tangential augmentation parameters and $\lambda$ a Lagrange multiplier. In Figure 2(right), we depict the traction-opening curve for a mixed linear non regularized cohesive law.

The effective cohesive traction is then given by $\boldsymbol{t}_{c}^{\prime}=\frac{\partial \Pi}{\partial \boldsymbol{w}}$. The additional dual equation, that corresponds to the interfacial law, is determined by stating that $\frac{\partial \Pi}{\partial \lambda}=0$.

The effective cohesive traction thus reads :

$$
\begin{aligned}
t_{c, n}^{\prime}\left(\lambda_{n}+r w_{n}, \lambda_{s}+r_{s} \boldsymbol{w}_{s}\right) & =\frac{\partial \Pi}{\partial w_{n}}=r \frac{\partial \psi}{\partial\left(\lambda_{n}+r w_{n}\right)} \\
\boldsymbol{t}_{c, s}^{\prime}\left(\lambda_{n}+r w_{n}, \lambda_{s}+r_{s} \boldsymbol{w}_{s}\right) & =\frac{\partial \Pi}{\partial \boldsymbol{w}_{s}}=r_{s} \frac{\partial \psi}{\partial\left(\lambda_{s}+r_{s} \boldsymbol{w}_{s}\right)}
\end{aligned}
$$

From here onward, we omit the dependence of $\boldsymbol{t}_{c}^{\prime}$ on $\lambda_{n}+r w_{n}$ and $\lambda_{s}+r_{s} \boldsymbol{w}_{s}$ to alleviate notations. The interfacial law simply reads $\lambda=\boldsymbol{t}_{c}^{\prime}$.

As suggested in [58], we introduce an equivalent augmented traction

$$
(\lambda+r w)_{\mathrm{eq}}:=\sqrt{\left\langle\lambda_{n}+r w_{n}\right\rangle_{+}^{2}+\frac{r}{r_{s}}\left(\lambda_{s}+r_{s} \boldsymbol{w}_{s}\right)^{2}}
$$

with $\left\langle\lambda_{n}+r w_{n}\right\rangle_{+}$the positive part of $\lambda_{n}+r w_{n}$ and a threshold function $\varphi$ such that :

$$
\varphi\left((\lambda+r w)_{\mathrm{eq}}\right):=\frac{(\lambda+r w)_{\mathrm{eq}}-\sigma_{c}}{r w_{c}-\sigma_{c}}
$$

with $w_{c}$ the critical displacement jump for the cohesive law, corresponding to the vanishing of the cohesive traction. A scalar dimensionless variable $\alpha$ is then defined as verifying :

$$
\begin{aligned}
\varphi\left((\lambda+r w)_{\mathrm{eq}}\right)-\alpha & \leq 0 \\
\dot{\alpha} & \geq 0 \\
\dot{\alpha}\left[\varphi\left((\lambda+r w)_{\mathrm{eq}}\right)-\alpha\right] & =0
\end{aligned}
$$

For an uncracked material, it holds $\alpha \leq 0$ and for a fully cracked material it holds $\alpha \geq 1$. For loading conditions, we have

$$
\left\{\begin{array}{l}
\dot{\alpha}>0 \\
\alpha=\varphi\left((\lambda+r w)_{\mathrm{eq}}\right)
\end{array}\right.
$$

and the function $\psi$ is defined by :

$$
\psi\left(\lambda_{n}+r w_{n}, \lambda_{s}+r_{s} \boldsymbol{w}_{s}\right)=2 G_{c}\left(1-\frac{\sigma_{c}}{r w_{c}}\right) \alpha\left(1-\frac{\alpha}{2}\right)+\frac{1}{2 r}\left\langle\lambda_{n}+r w_{n}\right\rangle_{-}^{2}
$$


with $\left\langle\lambda_{n}+r w_{n}\right\rangle_{-}$the negative part of $\lambda_{n}+r w_{n}$.

For contact-free situations (it then holds $\left\langle\lambda_{n}+r w_{n}\right\rangle_{-}=0$ ), the surface energy (13) depends only on $\alpha$ and $\lambda_{\text {eq }}$ : $\Pi\left(\alpha, \lambda_{\text {eq }}\right)=\psi(\alpha)-\frac{\lambda_{\mathrm{eq}}^{2}}{2 r}$. When the dissipation of energy starts, it holds :

$$
\left\{\begin{array}{l}
\alpha=0 \\
\lambda_{\text {eq }}=\sigma_{c}
\end{array}\right.
$$

and when it ends (breaking point), it holds

$$
\left\{\begin{array}{l}
\alpha=1 \\
\lambda_{\text {eq }}=0
\end{array}\right.
$$

Then, we have :

$$
\Pi\left(\alpha=1, \lambda_{\mathrm{eq}}=0\right)-\Pi\left(\alpha=0, \lambda_{\mathrm{eq}}=\sigma_{c}\right)=G_{c}
$$

which ensures that $G_{c}$ corresponds to the energy that shall be provided to fully debond a unit surface of fracture.

The resulting effective cohesive traction may be synthesized with an equivalent effective cohesive traction : $t_{c, e q}^{\prime}=$ $\sqrt{\left\langle t_{c, n}^{\prime}\right\rangle_{+}^{2}+\frac{r}{r_{s}} t_{c, s}^{\prime 2}}$, linked to the augmented cohesive traction via $: t_{c, \mathrm{eq}}^{\prime}=\left(1-T_{d}\right)(\lambda+r w)_{\mathrm{eq}}$ with $T_{d}$ the damage tensor :

$$
T_{d}=\frac{\alpha}{\left(1-\frac{\sigma_{c}}{r w_{c}}\right) \alpha+\frac{\sigma_{c}}{r w_{c}}}
$$

We satisfy $T_{d}=0$ if $\alpha=0$ (perfect adherence) and $T_{d}=1$ if $\alpha=1$ (breaking point). The general expression for the effective cohesive traction is :

$$
\begin{gathered}
t_{c, n}^{\prime}=\left(1-T_{d}\right)\left\langle\lambda_{n}+r w_{n}\right\rangle_{+}+\left\langle\lambda_{n}+r w_{n}\right\rangle_{-} \\
\boldsymbol{t}_{c, s}^{\prime}=\left(1-T_{d}\right)\left(\lambda_{s}+r_{s} \boldsymbol{w}_{s}\right)
\end{gathered}
$$

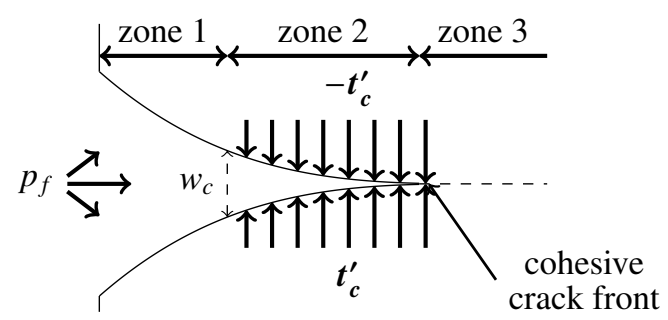

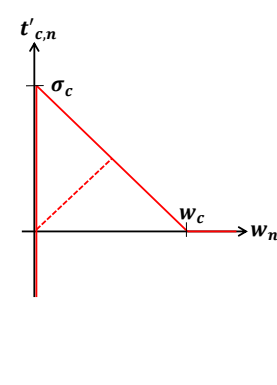

Opening mode

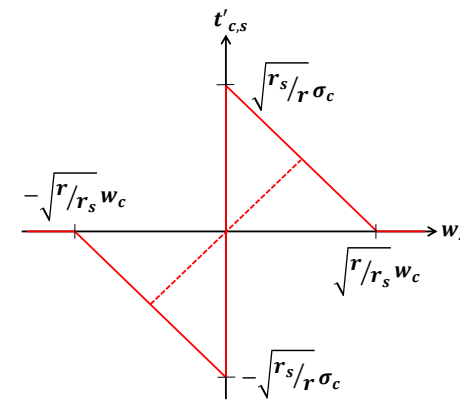

Shear mode

FIGURE 2: Representation of a cohesive zone model under fluid circulation (left) and the chart of a mixed linear cohesive law (right).

\subsubsection{Augmented Lagrangian}

As explained in [58], the quantities related to the interface (the cohesive traction $\boldsymbol{t}_{c}$ and the energy density $\Pi$ ) must be defined over a reduced space $M_{0}$ adapted to the cohesive fracture compared to the definition space used for the displacement field $U_{0}$. This is necessary in order to avoid spurious oscillations during adherence phases. $\boldsymbol{w}$ is introduced as a new unknown to the problem, defined over a different space from that of $\llbracket \boldsymbol{u} \rrbracket: M_{0}$. The construction of the approximation space for the fields defined over the reduced space $M_{0}$ is detailed in the next paragraph. The total energy of the domain $\Omega$ cut by the cohesive interface $\Gamma_{c}$ reads :

$$
E(\boldsymbol{u}, \lambda, \boldsymbol{w})=\frac{1}{2} \int_{\Omega} \boldsymbol{\varepsilon}(\boldsymbol{u}): \boldsymbol{C}: \boldsymbol{\varepsilon}(\boldsymbol{u}) d \Omega-\int_{\Gamma_{t}} \boldsymbol{t} \cdot \boldsymbol{u} d \Gamma_{t}+\int_{\Gamma_{c}} \Pi(\boldsymbol{w}, \lambda) d \Gamma_{c}-\int_{\Gamma_{c}} p_{f} \boldsymbol{n} \cdot \boldsymbol{w} d \Gamma_{c}
$$


The solution of the continuous problem implies to find $(\boldsymbol{u}, \boldsymbol{w}, \lambda)=\underset{\boldsymbol{w}^{*}=\llbracket \boldsymbol{u}^{*} \rrbracket}{\operatorname{argmin}} E\left(\boldsymbol{u}^{*}, \boldsymbol{w}^{*}, \lambda^{*}\right)$.

The Lagrangian of the problem is then :

$\mathscr{L}(\boldsymbol{u}, \boldsymbol{w}, \lambda, \boldsymbol{\mu})=\frac{1}{2} \int_{\Omega} \boldsymbol{\varepsilon}(\boldsymbol{u}): \boldsymbol{C}: \boldsymbol{\varepsilon}(\boldsymbol{u}) d \Omega-\int_{\Gamma_{t}} \boldsymbol{t} \cdot \boldsymbol{u} d \Gamma_{t}+\int_{\Gamma_{c}} \Pi(\boldsymbol{w}, \lambda) d \Gamma_{c}+\int_{\Gamma_{c}} \boldsymbol{\mu} \cdot(\llbracket \boldsymbol{u} \rrbracket-\boldsymbol{w}) d \Gamma_{c}-\int_{\Gamma_{c}} p_{f} \boldsymbol{n} \cdot \boldsymbol{w} d \Gamma_{c}$

with $\boldsymbol{\mu}$ a Lagrange multiplier introduced to enforce the condition $\llbracket \boldsymbol{u} \rrbracket=\boldsymbol{w}$ along the cohesive interface $\Gamma_{c}$. The Lagrange multipliers $\lambda$ and $\mu$, as well as the fluid pressure at the interface, are also defined over the reduced space $M_{0}$ adapted to the cohesive interface.

\subsubsection{Weak formulation of the mechanical problem}

The optimality conditions of this Lagrangian give the following discrete weak form :

$$
\left\{\begin{array}{l}
\forall \boldsymbol{u}^{*} \in \boldsymbol{U}_{0}, \int_{\Omega} \boldsymbol{\sigma}(\boldsymbol{u}): \boldsymbol{\varepsilon}\left(\boldsymbol{u}^{*}\right) d \Omega-\int_{\Gamma_{t}} \boldsymbol{t} \cdot \boldsymbol{u}^{*} d \Gamma_{t}+\int_{\Gamma_{c}} \boldsymbol{\mu} \cdot \llbracket \boldsymbol{u}^{*} \rrbracket d \Gamma_{c}=0 \\
\forall \boldsymbol{\mu}^{*} \in \boldsymbol{M}_{0}, \int_{\Gamma_{c}} \boldsymbol{\mu}^{*} \cdot(\llbracket \boldsymbol{u} \rrbracket-\boldsymbol{w}) d \Gamma_{c}=0 \\
\forall \boldsymbol{w}^{*} \in M_{0},-\int_{\Gamma_{c}} \boldsymbol{w}^{*} \cdot\left(\boldsymbol{\mu}+p_{f} \boldsymbol{n}-\boldsymbol{t}_{c}^{\prime}\right) d \Gamma_{c}=0 \\
\forall \lambda_{n}^{*} \in M_{0},-\int_{\Gamma_{c}} \frac{\lambda_{n}-t_{c, n}^{\prime}}{r} \lambda_{n}^{*} d \Gamma_{c}=0, \quad \forall \lambda_{s}^{*} \in M_{0},-\int_{\Gamma_{c}} \frac{\lambda_{s}-\boldsymbol{t}^{\prime}{ }_{c, s}}{r_{s}} \cdot \lambda_{s}^{*} d \Gamma_{c}=0
\end{array}\right.
$$

with : $\boldsymbol{U}_{0}=\left\{\boldsymbol{u}^{*} \in H^{1}(\Omega)\right.$ such that $\boldsymbol{u}^{*}$ discontinuous across $\Gamma_{c}$ and $\boldsymbol{u}^{*}=0$ on $\left.\Gamma_{u}\right\}$.

The first optimality condition represents the global mechanical equilibrium of the system. In particular, the term $\int_{\Gamma_{c}} \boldsymbol{\mu} \cdot \llbracket \boldsymbol{u}^{*} \rrbracket d \Gamma_{c}$ accounts for the cohesive efforts at the interface. The second optimality condition is interpreted as the projection of the displacement jump $\llbracket \boldsymbol{u} \rrbracket$ on the reduced space $M_{0}$. The third optimality condition is interpreted as the projection of the total cohesive traction $\boldsymbol{t}_{c}$ on the reduced space $M_{0}$. Finally, the fourth optimality condition is the interfacial law.

This new formulation for the cohesive zone model involves two additional degrees of freedom compared to the initial formulation presented in [34] : the displacement jump $\boldsymbol{w}$ and the Lagrange multiplier $\boldsymbol{\mu}$. Contrarily to what was done in [34], we do not proceed to collocations at the Gauss points of the cohesive interface. As already discussed, the displacements jump along the interface $\boldsymbol{w}$ is directly given at the vertex nodes of the intersected edges (it is a degree of freedom of the problem). The internal variables related to the cohesive fracture (essentially the rate of dissipated energy) shall then be stored at these vertex nodes. We make the same choice for the internal variables related to the fluid flow in the cohesive interface (essentially the density of the fluid $\rho$ in the interface).

\subsection{Discretization with XFEM}

\subsubsection{Fields related to the bulk}

The cohesive fracture $\Gamma_{c}$ in the domain $\Omega$ is implicitly defined by mean of two level-set functions [62], [63]. The first one is the normal level-set $(l s n)$ which gives the signed distance to the interface regardless of the crack front. The second one is the tangential level-set $(l s t)$ which gives the signed distance to the discontinuity front in the direction tangential to the crack surface. The fracture interface $\Gamma_{c}$ is then defined as the intersection between the surface $\{\boldsymbol{x} \in \Omega$ such that $l \operatorname{sn}(\boldsymbol{x})=0\}$ and the subdomain $\{\boldsymbol{x} \in \Omega$ such that $l s t(\boldsymbol{x})<0\}$ (see Figure 3 ). The discontinuity front is then the set of points : $\{\boldsymbol{x} \in \Omega$ such that $l \operatorname{sn}(\boldsymbol{x})=l s t(\boldsymbol{x})=0\}$. The level-set functions allow to implicitly locate the discontinuity surface independently from the mesh. And when the fracture propagates, it is only necessary to update the level-set functions. We made the choice to interpolate the level-set fields with the same shape functions as for the displacement field. This is a common choice which enables the fracture shape to be entirely described in terms of nodal values.

In order to simulate a potential displacement jump across a cohesive fracture $\Gamma_{c}$ in a domain $\Omega$, we introduce additional degrees of freedom $\boldsymbol{b}_{j}$ at the nodes $j$ whose support is intersected by the interface (see Figure 4). The sign of the normal level-set enables the distinction between two subdomains $\Omega_{+}$and $\Omega_{-}$on both sides of the interface such that $\Omega_{+} \cup \Omega_{-}=\Omega$ (see Figure 4). By applying the Heaviside function directly to the normal level-set, the discontinuity 

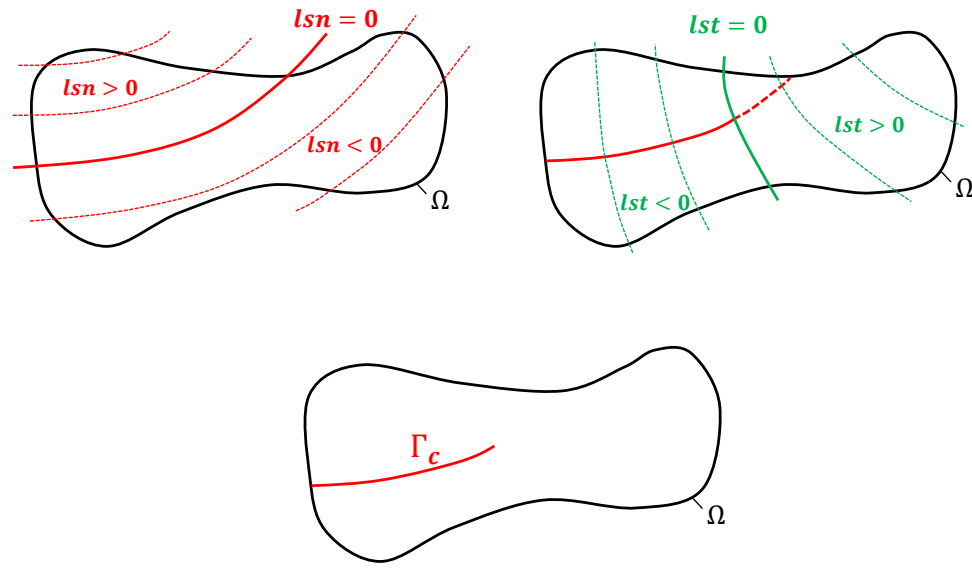

FIGURE 3: Normal level-set (left), tangential level-set (right) and the resulting fracture $\Gamma_{c}$ (bottom).

occurs at the interface. For a node $j$ whose position is denoted $\boldsymbol{x}_{j}$, the Heaviside function associated to the additional degree of freedom $\boldsymbol{b}_{j}$ is :

$$
\begin{aligned}
& \text { If } \boldsymbol{x}_{j} \in \Omega_{+}, H_{j}(l \sin (\boldsymbol{x}))=\left\{\begin{array}{r}
0 \text { if } l \sin (\boldsymbol{x})>0 \\
-2 \text { if } l \operatorname{sn}(\boldsymbol{x}) \leq 0
\end{array}\right. \\
& \text { If } \boldsymbol{x}_{j} \in \Omega_{-}, H_{j}(l \sin (\boldsymbol{x}))=\left\{\begin{array}{r}
0 \text { if } l \operatorname{ssn}(\boldsymbol{x})<0 \\
+2 \text { if } l \operatorname{sn}(\boldsymbol{x}) \geq 0
\end{array}\right.
\end{aligned}
$$
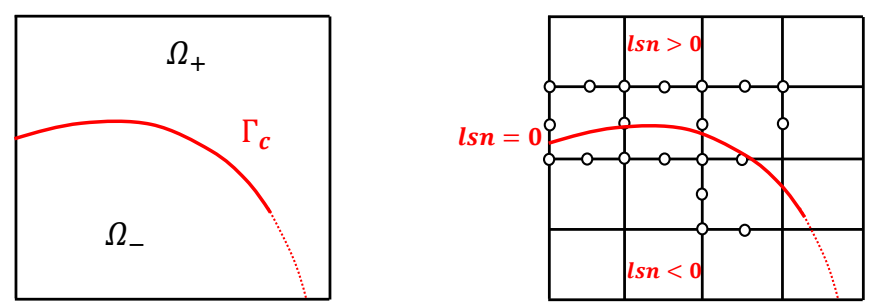

FIGURE 4: On the left, the two domains $\Omega_{+}$and $\Omega_{-}$formed by the arbitrary fracture $\Gamma_{c}$. On the right, the associated mesh. The surrounded nodes carry the Heaviside enrichment.

This definition depends on the position of the node $j$ compared to the interface $\Gamma$. The quantity $H_{j}(\operatorname{lsn}(\boldsymbol{x}))$ is 0 if the point $\boldsymbol{x}$ and the node $j$ are in the same subdomain and \pm 2 otherwise. The coefficient 2 is introduced in order to facilitate the expression of the displacement jump along the interface [56]. This "complementary" formulation has been introduced by Ndeffo [56] and is inspired by the work of Hansbo et al. [64]. Ndeffo proved that the deterioration in the conditioning of the problem is reduced by this "complementary" formulation compared with the formulation of Moës et al. [22].

As mentioned in the introduction, the displacement field is interpolated in a quadratic way and the pore pressure field is interpolated in a linear way. The approximation of the displacement field is then :

$$
\boldsymbol{u}_{h}(\boldsymbol{x})=\sum_{i \in N} \boldsymbol{a}_{i} \psi_{i}(\boldsymbol{x})+\sum_{j \in N \cap N_{H}} \boldsymbol{b}_{j} \psi_{j}(\boldsymbol{x}) H_{j}(l \operatorname{sn}(\boldsymbol{x}))
$$

where $N$ is the set of nodes whose support contains the point $\boldsymbol{x}$ and $N_{H}$ the set of nodes which carries the Heaviside enrichment. $\boldsymbol{a}_{i}$ are the nodal values for the classical part of the displacement field and $\boldsymbol{b}_{j}$ are the nodal values for the enriched part of the displacement field. $\psi_{i}$ are the quadratic Lagrange polynomial shape functions. 
The approximation of the pore pressure field is :

$$
p_{h}(\boldsymbol{x})=\sum_{i \in N} c_{i} \phi_{i}(\boldsymbol{x})+\sum_{j \in N \cap N_{H}} d_{j} \phi_{j}(\boldsymbol{x}) H_{j}(l s n(\boldsymbol{x}))
$$

where $N$ is the set of vertex nodes whose support contains the point $\boldsymbol{x}$ and $N_{H}$ the set of vertex nodes which carries the Heaviside enrichment. $c_{i}$ are the nodal values for the classical part of the pore pressure field and $d_{j}$ are the nodal values for the enriched part of the pore pressure field. $\phi_{i}$ are the linear Lagrange polynomial shape functions.

\subsubsection{Fields related to the fracture}

In Figure 5, we depict a quadrangular HM-XFEM element and its associated degrees of freedom.

We recall that for the fields related to the cohesive fracture $\Gamma_{c}\left(p_{f}, q_{+}, q_{-}, \lambda, \boldsymbol{\mu}, \boldsymbol{w}\right)$, the set of admissible functions is :

$$
M_{0}=\left\{\Lambda^{*} \in H^{-\frac{1}{2}}\left(\Gamma_{c}\right)\right\}
$$

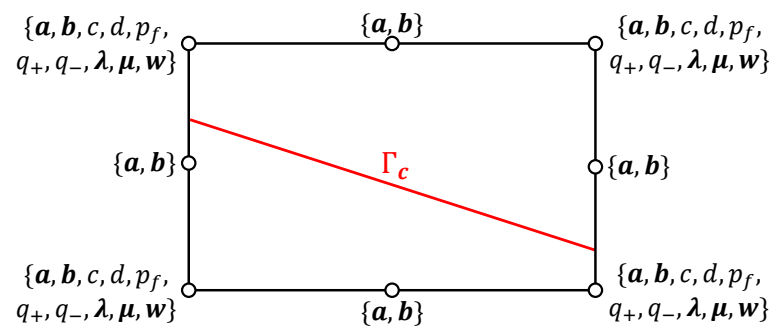

FIGURE 5: A quadrangle cohesive HM-XFEM element.

The approximation space for the fields related to the cohesive fracture is then adapted to the fracture path. It relies on the vertex nodes of the edges intersected by the discontinuity $\Gamma_{c}$. As explained in [34], the approximation space is reduced in order to satisfy the LBB stability condition [23] and avoid the appearance of spurious oscillations in the numerical resolution. For this aim, we impose equality relationships across the discontinuity for fields related to the cohesive interface. This approach was first suggested by Moës et al. [65] and then improved by Béchet [66]. Based on the work of Moës et al. [65], Géniaut developed a specific algorithm for the selection of edges carrying those equality relationships [67] based on the concept of vital edges. In the first instance, we set up groups with the intersected edges that share a common vertex node. Any edge tagged in a group is considered vital. Then, if an edge of a group can be removed without creating an orphan vertex node, it is considered as non vital. Finally, equality relationships are prescribed between the cohesive degrees of freedom of the vertex nodes of the remaining vital edges. So, for a group of connected vital edges, a cohesive field $\Lambda$ is approximated only by a single and common degree of freedom. In Figure 6 on top, we depict an example for an interface that crosses a triangular mesh. Vital edges are dashed, and for a cohesive field $\Lambda$, the dimension of the approximation space is 4 , which corresponds to the number of groups of vital edges.

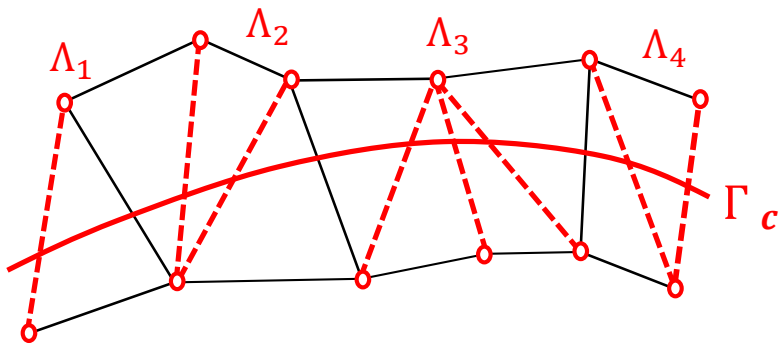

FIGURE 6: Approximation space for the fields related to the cohesive fracture. 


\section{Validation of the mortar HM-XFEM model on the penny-shaped benchmark}

In order to validate this new model, we perform a comparison with an analytical solution that involves a plane fracture in a $3 \mathrm{D}$ poro-elastic medium. This test is known as the penny shaped hydraulic fracture [8] and the corresponding analytical asymptotic solution is detailed in [68].

\subsection{The penny-shaped model}

The geometry of the problem is represented in Figure 7 A constant fluid flux $Q_{0}$ is injected punctually in a poroelastic semi-infinite saturated medium, subject to a vertical confining stress $\sigma_{0}$. The injected fluid is incompressible. We simulate the propagation of a radial horizontal hydraulic fracture from the injection point, in the plane perpendicular to the injection well. Note that the analytical solution of the asymptotic behavior studied by [68] relies on the following assumptions : (i) deformation of the elastic solid matrix due to the rise in the fluid pressure applied on the fracture walls, (ii) fluid flow in the fracture is governed by the cubic law, (iii) the leak-off is supposed to be one-dimensional in the direction perpendicular to the plane surface and given by Carter's law [52] and (iv) fracture propagation is in mobile equilibrium.

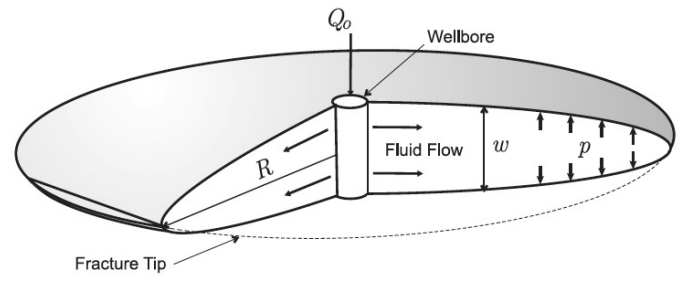

FIGURE 7: Penny shaped model (from 69)

\subsection{Geometry and boundary conditions}

In order to simulate the penny-shaped model, we consider a cylinder of height $H=15 \mathrm{~m}$ and radius $R=9 \mathrm{~m}$. The cylinder is crossed at mid-height by a planar cohesive interface represented by means of a normal level-set only. We do not use a tangential level-set as the cylinder is supposed to be entirely crossed by the potential crack surface. Under the effects of the fluid injection at the center of the potential crack surface, a radial fracture expands. The cohesive zone model naturally separates the opened zone from the adherent zone. The pore pressure is set to zero at the top and at the bottom of the cylinder.

The material properties for the solid matrix, for the fluid and for the cohesive zone model are summarized in Table 1.

\begin{tabular}{|c|c|}
\hline Young's modulus & $E=17 G P a$ \\
\hline Poisson's ratio & $v=0.2$ \\
\hline Biot's coefficient & $b=0.75$ \\
\hline Intrinsic permeability & $k^{i n t}=10^{-16} m^{2}$ \\
\hline Porosity & $\phi=0.2$ \\
\hline Dynamic viscosity & $\mu=10^{-4}$ Pa.s \\
\hline Compressibility modulus of the fluid & $\frac{1}{K_{w}}=0 P a^{-1}$ \\
\hline Density of the fluid & $\rho=1000 \mathrm{~kg} \cdot \mathrm{m}^{-3}$ \\
\hline Critical stress & $\sigma_{c}=1.25 \mathrm{MPa}$ \\
\hline Cohesive energy & $G_{c}=200 P a . m$ \\
\hline Augmentation parameters & $r=r_{s}=2$ \\
\hline
\end{tabular}

TABLE 1: Material parameters for the porous matrix and the fluid.

The mesh we use is constituted of 36234 hexahedra and 366 pentahedra (see Figure 8). The problem has a cylindrical symmetry, so that we only consider an angular fraction of the domain $\left(15^{\circ}\right.$ of angle). The normal displacements are set to zero on the lateral faces. The mesh is refined in the vicinity of the cohesive interface. 

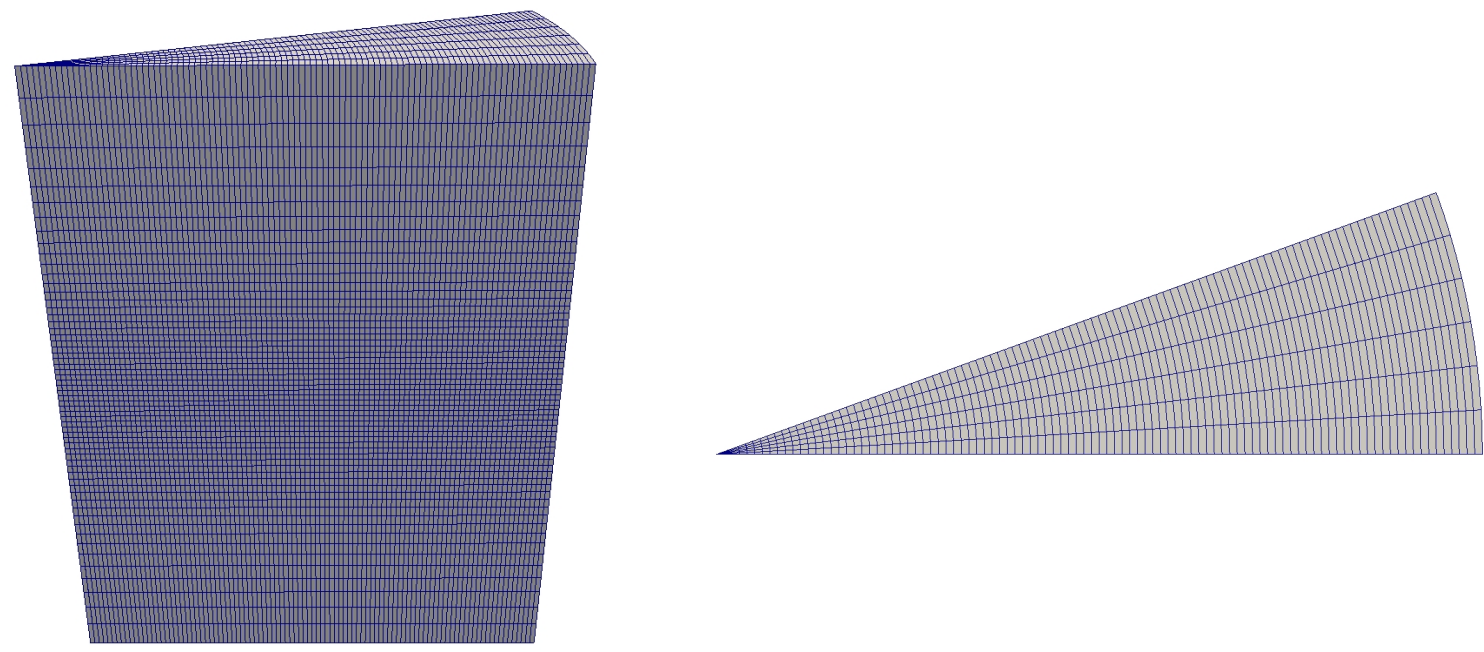

FIGURE 8: Mesh used for the penny shaped test : lateral side (left) and top side (right).

We inject the fluid with a constant flow rate $Q_{0}=3 \mathrm{~kg}_{\mathrm{s}} \mathrm{s}^{-1}$ at the center of the crack fracture surface for 8 seconds (see Figure 9) and we impose a vertical confining stress $\sigma_{0}=3.7 \mathrm{MPa}$. The equivalent leak-off coefficient for Carter's law $C_{L}$ is determined a posteriori. We found it equal to $2.7 \cdot 10^{-5} \mathrm{~m} \cdot \mathrm{s}^{-\frac{1}{2}}$.

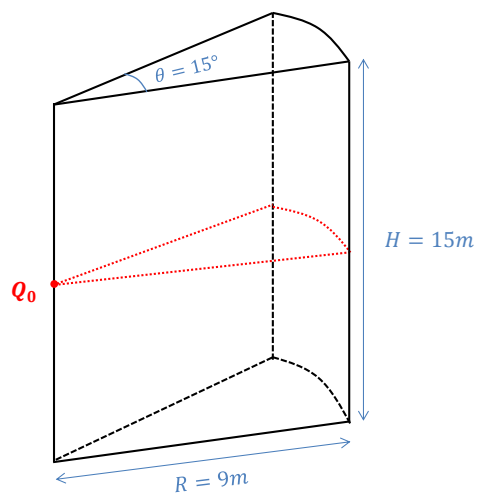

FIGURE 9: Geometry for the penny-shaped benchmark test.

\subsection{Analytical model}

According to Bunger et al. [68], two energy dissipation mechanisms compete while the hydraulic fracture develops : the creation of surface area and the viscous flow dissipation. Two asymptotic propagation regimes may then be distinguished : the toughness dominated regime and the viscosity dominated regime. Bunger et al. [68] have then introduced the time independent dimensionless parameter $\mathscr{M}_{k}$ to characterize the predominance of the dissipation mechanism. It can be interpreted as a dimensionless viscosity :

$$
\mathscr{M}_{k}=12 \mu\left(\frac{C^{\prime 4} E^{11} Q_{0}}{K^{\prime 14}}\right)^{\frac{1}{3}}
$$

with $K^{\prime}=4\left(\frac{2}{\pi}\right)^{\frac{1}{2}} \sqrt{E G_{c}}$ and $C^{\prime}=2 C_{L}$ the leak-off coefficient.

Simultaneously, the storage of fluid in the fracture compete with the leak-off phenomenon. At early times, most of the fluid is stored in the opened crack. But the relative importance of the leak-off phenomenon increases as the 
injection continues [68]. The competition between these two mechanisms can be appreciated in the light of a second dimensionless parameter, the dimensionless time $t^{*}$ :

$$
t^{*}=\left(\frac{K^{\prime 4} Q_{0}}{C^{\prime 5} E^{4}}\right)^{\frac{2}{3}}
$$

For small time scales (i.e. $t<<t^{*}$ ), the storage mechanism dominates and for large time scales (i.e. $t>>t^{*}$ ), the leak-off phenomenon dominates. With the set of parameters we selected, $\mathscr{M}_{k} \simeq 2.9 \cdot 10^{-3} \ll 1$. As explained in [68], under such circumstances, the propagation regime is governed by the toughness of the solid matrix and the solution of the problem only depends on the dimensionless time $t^{*}$. In our case, $t^{*} \sim 2.10^{5} \mathrm{~s}$. Small and large time asymptotic solutions are derived in [68], which correspond to regimes dominated by storage of fluid in the fracture and infiltration of fluid into the rock, respectively. It is obtained with a scaling and then an expansion of the constitutive equations of the model. In particular, the solution gives a singularity of $\frac{1}{2}$ (i.e $w \sim r^{\frac{1}{2}}, w$ being the aperture and $r$ being the distance to the crack front) in the vicinity of the crack tip. In the present work, we compare the toughness-dominated regime solution detailed in [68] to the numerical results we obtain at $t=8 \mathrm{~s}$.

\subsection{Numerical results}

\subsubsection{Fracture profile}

The amplified deformed shape and the pore pressure obtained at $t=8 \mathrm{~s}$ are represented in Figure 10 left. The solution possesses the radial symmetry. For a fixed radius $r$, the relative variations in the solution along the arc length are less than $0.3 \%$.
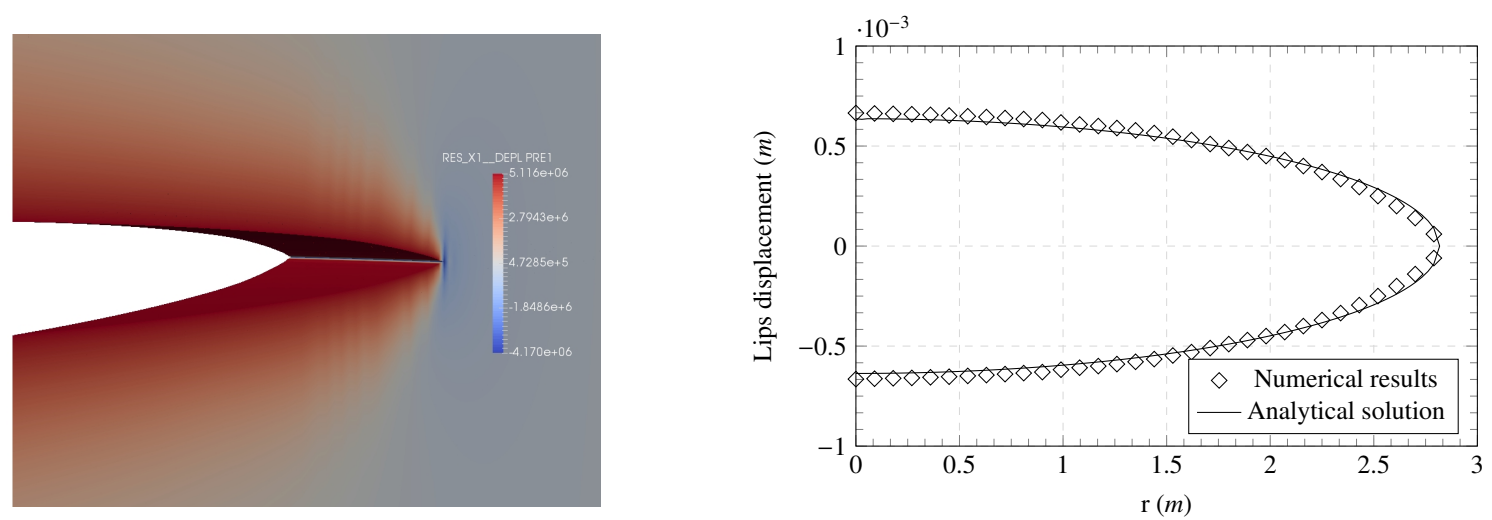

FIGURE 10: Amplified deformed shape (X1000) and pore pressure in $P a$ (left) and fracture profile (right) at $t=8 s$.

The agreement with the analytical solution is satisfactory and is comparable to the results obtained in the 2D case [34]. Our HM-XFEM model succeeds in giving an accurate representation of the fracture profile predicted by the theoretical model in the toughness dominated regime.

\subsubsection{Equivalent leak-off coefficient}

In the penny shaped hydraulic fracture model, the fluid losses from the fracture to the porous matrix are simulated by Carter's law [52]. The fluid losses by unit of surface area $g(r, t)$ are then :

$$
g(r, t)=\frac{2 C_{L}}{\sqrt{t-t_{0}(r)}}
$$

where $t_{0}(r)$ is the time it takes for the fluid-driven fracture to reach the radius $r$ and $C_{L}$ is the leak-off coefficient (the coefficient 2 in (36) is added to account for both fracture walls). In our HM-XFEM numerical model, the hydromechanical coupling between the porous matrix and the fracture is total. The mass exchanges between the hydraulic fracture and the porous matrix (degrees of freedom $q_{+}$and $q_{-}$) are obtained after the resolution of the coupled problem. We 
propose to verify the adequacy of Carter's law with our fully coupled hydromechanical model. In particular, we determine a posteriori an equivalent leak-off coefficient $C_{L}$. For this aim, we select a position at radius $r=1 \mathrm{~m}$ and we determine the time $t_{0}(r)$ it takes for the fracture to reach this radius. Then, we note the values of $q_{+}$and $q_{-}$at this radius for different values of $t$ larger than $t_{0}(r)$. The problem being symmetrical with respect to the fracture plane, we check that $q_{+}$and $q_{-}$are equal. We depict $q_{+}$, the quantity of fluid transiting from the fracture to the upper part of the porous matrix, with respect to $\frac{1}{\sqrt{t-t_{0}(r)}}$. The graph obtained is represented in Figure 11 .

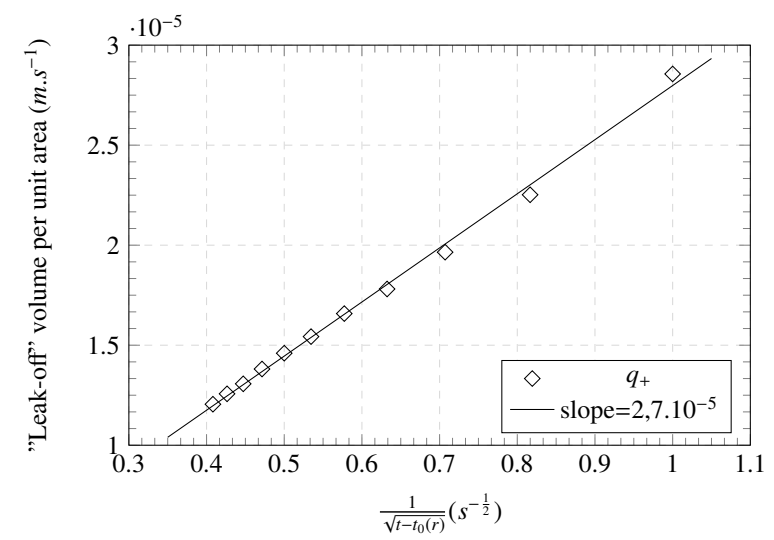

FIGURE 11: Determination of the leak-off coefficient $C_{L}$ at $r=1 \mathrm{~m}$.

The values of $q_{+}$obtained are satisfactorily aligned. Carter's law seems adapted to simulate the leak-off in our fully coupled hydromechanical model. The associated leak-off coefficient is $C_{L}=2.7 \cdot 10^{-5} \mathrm{~m} \cdot \mathrm{s}^{-\frac{1}{2}}$. We performed the same experience for a different position $(r=1.5 m)$ and found the same value for $C_{L}$.

The match between Carter's law and our fully coupled hydromechanical model is obviously not systematically verified. The $2 \mathrm{D}$ diffusion pattern assumed in Carter's model remains valid under the assumption that the fluid filled region is small compared to the crack length. Also, the limitations of Carter's law for large time scales have been highlighted in [70]. And once the fracture path is not planar or when several nearby hydraulic fractures interact, Carter's law is inapplicable. It is then a major advantage to dispose of a fully coupled hydromechanical model to simulate the leak-off.

\section{Fracture propagation on non-predefined paths}

In this section, we present the mathematical procedure for the $3 \mathrm{D}$ propagation of fluid-driven fractures on non predefined paths. The crack advance is still controlled by the cohesive zone model, but the bifurcation of the fracture path ahead of the crack front is determined by post-processing the cohesive state. Within the mesh, the propagation of the fractures corresponds to an update of the level-set fields. Examples involving nearby competing fluid-driven fractures and a $\mathrm{D}$ fracture reorientation case are presented.

\subsection{Overview of the procedure}

The strategy we adopt for the propagation on non-predefined paths has been developed by Ferté [58] to study the propagation of fractures in concrete. It is based on the cohesive zone model described in paragraph 2.3 The originality of this procedure lies in the computation of the fracture bifurcation angle based on the cohesive state. In practice, the crack advance is performed with an update of the level-set fields with a geometrical moving front algorithm [62], [71], [72].

In our model, the simulation of the propagation of a fluid-driven crack on a predefined path only requires a normal level-set field, whose iso-zero represents the crack path. Along this discontinuity surface, the cohesive zone model allows to distinguish the adherent zone from the debonding zones. In order to set up the propagation of a fluiddriven crack on a non-predefined path, the crack front must be implicitly located so that the cracked domain may 
be partitioned from the uncracked domain. In this section the cohesive fractures are then systematically defined with both a normal level-set field and a tangential level-set field. The discontinuity front correspond to the intersection of the iso-zeros of both level-set fields. We shall then distinguish the discontinuity front from the cohesive crack front that separates the adherent from the debonding zones. The discontinuity surface defined with the level-sets by : $\{\boldsymbol{x} \in \Omega: l s n(\boldsymbol{x})=0 \cap l s t(\boldsymbol{x})<0\}$ is interpreted as a potential crack surface. The actual fracture lies on the potential crack surface. It is delineated by the cohesive crack front (see Figure 12 top left). The area located between the cohesive crack front and the discontinuity front end is the adherent zone, that is subject to reorientations. The procedure for the propagation of fractures on non predefined paths is summarized in Figure 12
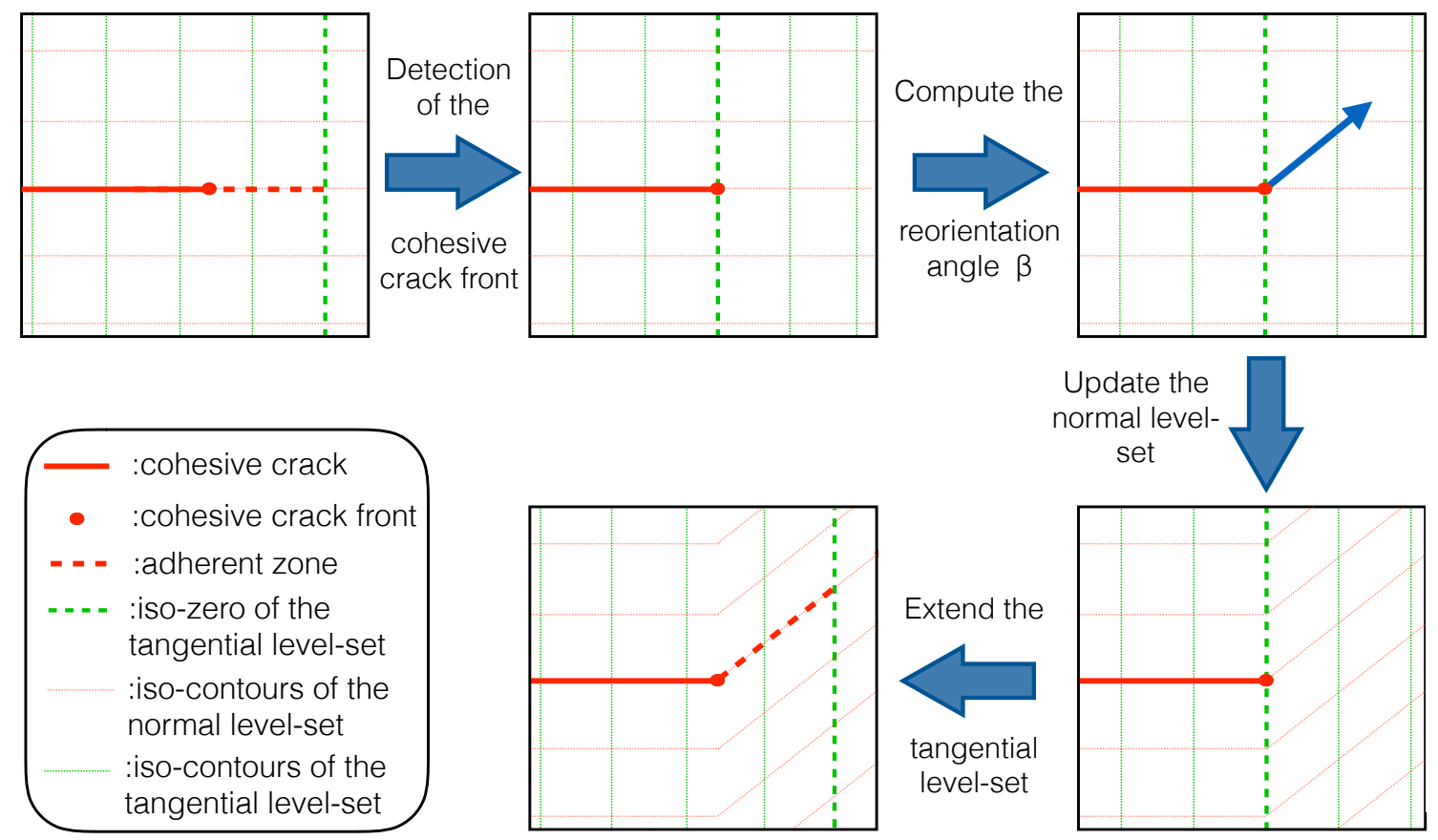

FIGURE 12: Overview of the procedure for the propagation of cohesive fluid-driven fractures on non-predefined paths.

We consider an initial potential crack surface defined with a normal level-set field and a tangential level-set field. In this potential crack surface, the cohesive crack front separates the cracked surface from the adherent zone (Figure 12 top left). We first proceed to the detection of the cohesive crack front and update the tangential level-set so that the discontinuity front coincides with the cohesive crack front (Figure 12 top middle). In this way, the half-space $\{\boldsymbol{x} \in \Omega: \operatorname{lst}(\boldsymbol{x})<0\}$ delineates the area where the normal level-set needs to be updated. Then we compute the bifurcation angle $\beta$ (see Figure 12 top right) and update the normal level-set field in accordance with this angle ahead of the crack front (Figure 12 bottom right), in the domain $\{x \in \Omega: l s t(x)<0\}$. Finally, we extend the potential crack surface (Figure 12 bottom middle). Lastly, we apply an orthogonalization procedure for the tangential level-set.

Remark : for the phases involving the establishment of the mechanical equilibrium, the potential crack surface must be taken sufficiently extended to prevent the actual cohesive crack from hitting the discontinuity front end. For this aim, we always take a margin in the definition of the potential crack surface, and once the update of the level-sets has been performed, we extend the potential crack surface (Figure 12 bottom middle) to prepare the next step. In practice, the extension of the potential crack surface is none other than a translation of the tangential level-set field.

\subsection{Detection of the cohesive crack front}

As previously explained in paragraph 2.3 the internal variables associated to the cohesive interface are carried by the vertex nodes of the edges cut by the discontinuity. In particular, we dispose of the internal variable $\alpha$ for this set of vertex nodes. This internal variable quantifies the dissipated energy in the cohesive zone. It satisfies $\alpha \leq 0$ in the adherent zone (where the damage process has not started) and $0<\alpha<1$ in the process zone. In the opened zone, we 
have $\alpha=1$ (all the cohesive energy has been dissipated). We use this internal variable for the detection of the cohesive crack front, that corresponds to the iso-zero of $\alpha$, i.e. the limit between the adherent zone and the process zone. Within the mesh, the cohesive crack front is then found as the intersection between the iso-zero of the normal level-set field and the iso-zero of the internal variable $\alpha$. We determine the intersection points between the cohesive crack front and the faces of the mesh with a Newton-Raphson algorithm. The chain of points we obtain is an approximation of the cohesive crack front. Finally, we update the tangential level-set field with a geometrical moving front algorithm, in order to merge the cohesive crack front and the discontinuity front end. The details of the moving front algorithm can be found in [71]. An exemple is represented in Figure 13 for a plane fracture embedded in a hexahedral mesh.

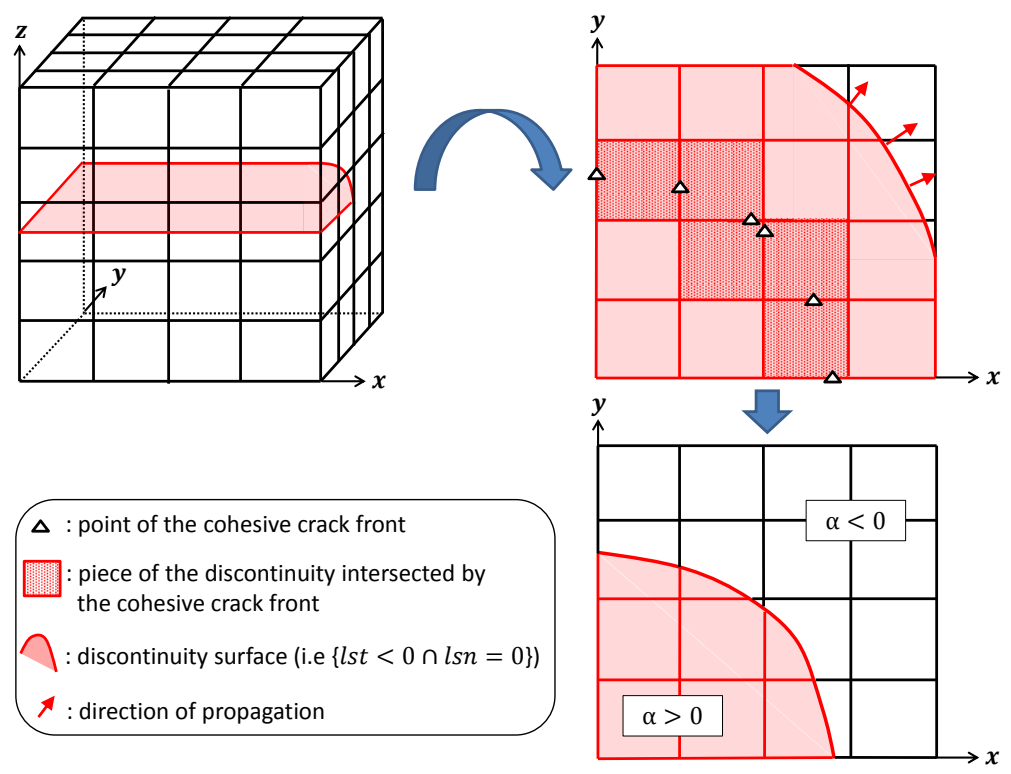

FIGURE 13: Detection of the cohesive crack front and update of the tangential level-set.

\subsection{Bifurcation angle}

Amongst the criteria for the bifurcation of fractures, we favor a criterion that rely on global quantities rather than local fields, in order to generate smooth crack fronts. We choose the maximum hoop stress criterion of Erdogan and Sih [73], written in terms of stress intensity factors :

$$
\beta=2 \arctan \left[\frac{1}{4}\left(K_{I} / K_{I I}-\operatorname{sign}\left(K_{I I}\right) \sqrt{\left(K_{I} / K_{I I}\right)^{2}+8}\right)\right]
$$

It consists in finding the direction of the maximum hoop stress. The reoriented fracture path is then assumed perpendicular to the maximum hoop stress direction.

Remark : for three-dimensional problems, the crack front is a curve. It is approximated by a chain of segments connecting the intersection points between the cohesive crack front and the faces of the elements of the mesh (see paragraph 4.2). A given number of calculation points are then distributed over this chain of segment. The stress intensity factors as well as the bifurcation angle $\beta$ are computed at these calculation points. Finally, $\beta$ is interpolated over the crack front from the values computed at the calculation points.

In order to determine the stress intensity factors, we use the method proposed by Ferté [58]. Ferté has demonstrated that a $J$-integral may still be defined in the context of cohesive zone models and it is expressed in terms of a cohesive integral only :

$$
J=-\int_{\Gamma_{c}} \boldsymbol{t}_{c} \cdot \nabla \llbracket \boldsymbol{u} \rrbracket \cdot \boldsymbol{\theta} d \Gamma_{c}
$$

with $\llbracket \boldsymbol{u} \rrbracket$ the displacement jump across the cohesive fracture, $\boldsymbol{t}_{c}$ the total cohesive traction applied on the fracture walls and $\boldsymbol{\theta}$ a virtual extension of the fracture (see Figure 14), that is a field of unit vectors tangent to the fracture surface pointing towards the crack front. 


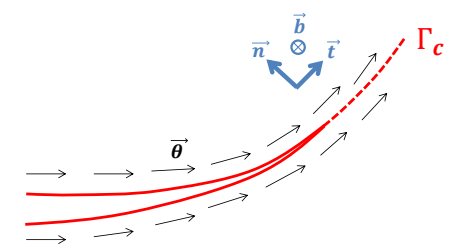

FIGURE 14: Virtual extension of the cohesive fracture $\Gamma_{c}$.

Ferté also established that this Rice cohesive integral is equal to the quantity $\mathrm{G}$ (derivative of the total potential energy of the structure with respect to the crack advance), expressed in terms of the stress intensity factors with Irwin's formula :

$$
J=G=\frac{1-v^{2}}{E}\left(K_{I}^{2}+K_{I I}^{2}\right)+\frac{1}{2 \mu} K_{I I I}^{2}
$$

In the context of Biot effective stress, for our hydromechanical problem we have : $\boldsymbol{t}_{c}=\boldsymbol{t}^{\prime}{ }_{c}-p_{f} \boldsymbol{n}=\left(t_{c, n}^{\prime}-p_{f}\right) \boldsymbol{n}+$ $t_{c, t}^{\prime} \boldsymbol{t}+t_{c, b}^{\prime} \boldsymbol{b}$. Furthermore, $\boldsymbol{\nabla} \llbracket \boldsymbol{u} \rrbracket \cdot \boldsymbol{\theta}=(\boldsymbol{n} \cdot \boldsymbol{\nabla} \llbracket \boldsymbol{u} \rrbracket \cdot \boldsymbol{\theta}) \boldsymbol{n}+(\boldsymbol{t} \cdot \boldsymbol{\nabla} \llbracket \boldsymbol{u} \rrbracket \cdot \boldsymbol{\theta}) \boldsymbol{t}+(\boldsymbol{b} \cdot \boldsymbol{\nabla} \llbracket \boldsymbol{u} \rrbracket \cdot \boldsymbol{\theta}) \boldsymbol{b}$. Finally, combining equations (38) and (39), it comes :

$$
\begin{gathered}
K_{I}^{2}=-\frac{E}{1-v^{2}} \int_{\Gamma_{c}}(\boldsymbol{n} \cdot \boldsymbol{\nabla} \llbracket \boldsymbol{u} \rrbracket \cdot \boldsymbol{\theta})\left(t_{c, n}^{\prime}-p_{f}\right) d \Gamma_{c} \\
K_{I I}^{2}=-\frac{E}{1-v^{2}} \int_{\Gamma_{c}}(\boldsymbol{t} \cdot \boldsymbol{\nabla} \llbracket \boldsymbol{u} \rrbracket \cdot \boldsymbol{\theta}) t_{c, t}^{\prime} d \Gamma_{c} \\
K_{I I I}^{2}=-2 \mu \int_{\Gamma_{c}}(\boldsymbol{b} \cdot \boldsymbol{\nabla} \llbracket \boldsymbol{u} \rrbracket \cdot \boldsymbol{\theta}) t_{c, b}^{\prime} d \Gamma_{c}
\end{gathered}
$$

Thus, from the computation of cohesive integrals only, we determine the stress intensity factors $K_{I}$ and $K_{I I}$ needed to compute the bifurcation angle $\beta$.

Remark : in this paragraph, we transposed the results established by Ferte [58] in the context of brittle fracture for homogeneous isotropic elastic media to our hydromechanical problem. However, we must note that the preliminary assumptions necessary to the establishment of these results are not valid anymore in the hydromechanical context, notably because of the viscosity of the fluid. We must also note that the formula used for the determination of the bifurcation angle $\beta$ accordingly to the maximum hoop stress criterion remains valid only when the singularity in the crack tip profile is $\frac{1}{2}$. This is the case for toughness-dominated regimes but not in the general case. For instance, viscosity-dominated regimes involve a singularity $\frac{1}{3}$ in the crack tip profile [4]. Nonetheless, we should keep in mind that these calculus lead to the determination of the bifurcation angle $\beta$ only. What is crucial for the bifurcation is to operate a reorientation in the direction normal to the minimum confining stress. The choice was made to keep on using the method developed by Ferté for the propagation of fractures on non predefined paths. In addition, the criterion suggested by Ferté is practical in the sense that it relies on global energetic quantities appealing to cohesive integrals only.

Once we have determined the bifurcation angle $\beta$, we proceed to the update of the normal level-set field. The normal level-set field is updated from the detected cohesive crack front with a geometrical algorithm [71]. The cracked surface must remain unchanged. To this aim, the update of the normal level-set field is performed only in the domain $\{\boldsymbol{x}: l s t(\boldsymbol{x})>0\}$ (the iso-zero of the tangential level-set fits the position of the cohesive crack front at this stage).

\subsection{Applications}

In this paragraph, we perform three numerical tests involving the propagation of fluid-driven fractures on non predefined paths. Note that simulations on similar configurations have been carried out by Wang [48, 49] and will be used for qualitative comparison purpose. In the first place, we make two elementary tests of parallel fractures in a square plate to observe the mutual influence of two nearby fluid-driven fractures. The first one concerns two parallel fractures on the same side and the second one concerns two opposite fractures. These two tests constitute a basis to study the competition that takes place in a fluid-driven fracture network. Finally, we study the reorientation of a three-dimensional fracture depending on the regional stress. With the set of parameters we selected for the three tests, the dimensionless viscosity $\mathscr{M}_{k}$ is around $10^{-3}$ so that we can assume toughness-dominated propagation regimes. 


\subsubsection{Two parallel fluid-driven fractures}

This first test focuses on the competition between two parallel fluid-driven fractures propagating in the same direction. We consider a square plate of side $L=10 \mathrm{~m}$. The vertical displacements are blocked on the lower and upper edges and the horizontal displacements are blocked on the lateral edges. We introduce two vertical potential crack surfaces intersecting the lower edge of the square bar and distant of $d=3 m$ (see Figure 15 left). We inject the fluid with a constant flow rate $Q=3.10^{-1} \mathrm{~kg} \cdot \mathrm{s}^{-1}$ at both inlets for a total duration of $16 \mathrm{~s}$. The time step $\Delta t$ we use is $0.1 \mathrm{~s}$ during the first second and $0.25 \mathrm{~s}$ then. This choice will be discussed in section 4.4.4 We use a regular mesh, made of 1600 quadrangles. The material characteristics are summarized in Table 2

\begin{tabular}{|l|l|}
\hline Young's modulus & $E=5800 \mathrm{MPa}$ \\
Poisson ratio & $v=0.2$ \\
Density of the fluid & $\rho=1000 \mathrm{~kg} . \mathrm{m}^{-3}$ \\
Biot coefficient & $b=0.8$ \\
Intrinsic permeability & $k^{\text {int }}=10^{-15} \mathrm{~m}^{2}$ \\
Viscosity of the fluid & $\mu=0.001 \mathrm{~Pa} . \mathrm{s}$ \\
Porosity & $\phi=0.1$ \\
Compressibility modulus of the fluid & $\frac{1}{K_{w}}=5 \cdot 10^{-10} \mathrm{~Pa}^{-1}$ \\
Critical stress & $\sigma_{c}=1 \mathrm{MPa}$ \\
Cohesive energy & $G_{c}=900 \mathrm{~Pa} . \mathrm{m}$ \\
Augmentation parameters & $r=r_{s}=2$ \\
\hline
\end{tabular}

TABLE 2: Material parameters for the porous medium and the fluid

For the first 6 seconds, we do not update the potential crack surfaces, so that both fractures are forced to propagate in the vertical direction. Between $t=6 \mathrm{~s}$ and $t=16 \mathrm{~s}$, we update the potential crack surfaces each second. This choice for the frequency of the updates is justified by the sensitivity analysis conducted in section 4.4.4 in order to reduce computational time without loss on the accuracy of predicted trajectories.

The amplified deformed shape and the pore pressure obtained at $t=16 \mathrm{~s}$ are represented Figure 15 (right).
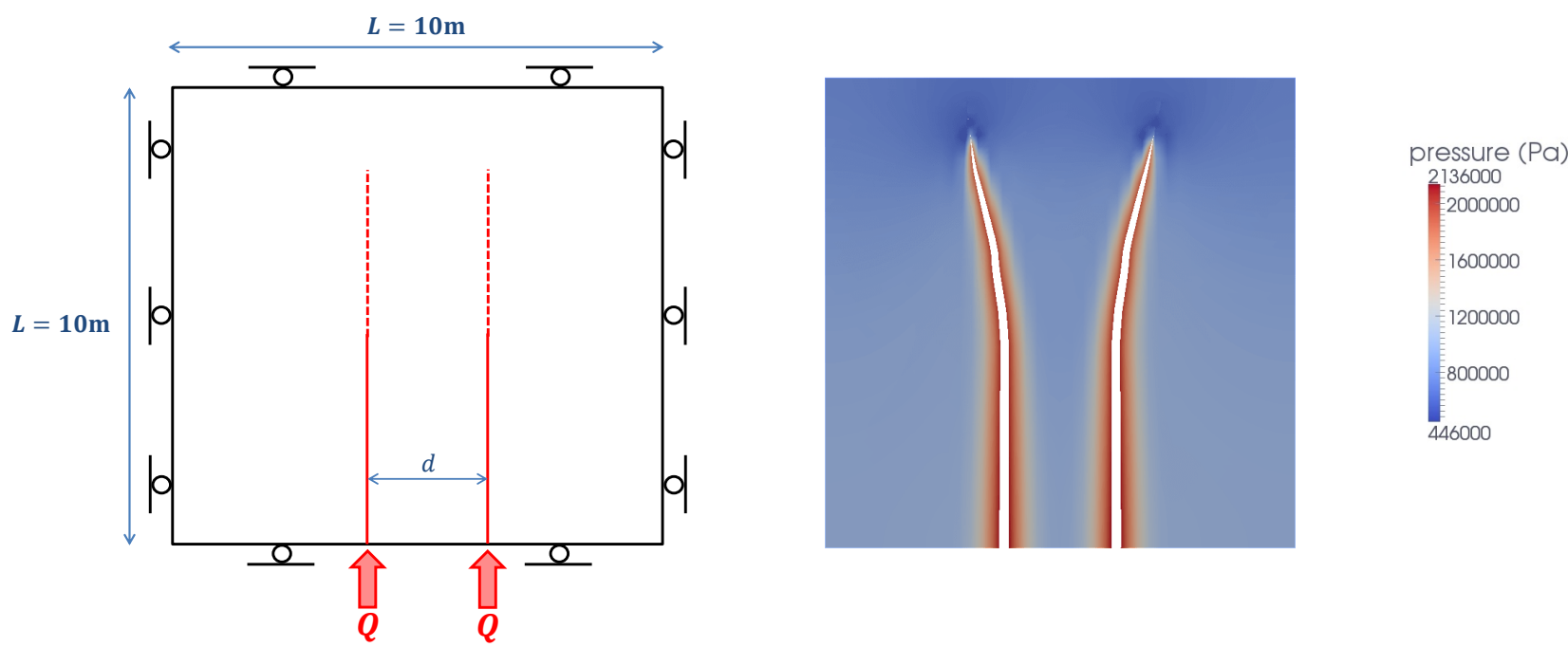

FIGURE 15: Two parallel fluid-driven fractures : geometry of the problem (left), amplified deformed shape (X100) and pore pressure at $t=16 \mathrm{~s}$ (right).

The two fluid-driven fractures expand vertically during the first 6 seconds. As we start to apply the fracture reorientation procedure each second, their trajectories diverge. At $t=16 \mathrm{~s}$, the aperture angle between the two fracture tips is around $17^{\circ}$. Furthermore, the pattern we get is symmetric, as the two fluid-driven fractures behave similarly. 
When fractures are propagating, the pore fluid pressure increases more specifically between fractures and induces a disturbance in the stress field in the crack tip areas [49]. The presence of a nearby fracture on the right side of the left fracture induces a dissymmetry in the stress field faced by the left fracture and vice versa. The two fractures have the tendency to pull over, continuing their extension in the direction of least resistance, i.e. of the maximal confining stress. They pull away in order to stop impeding each other. Results are physically consistent and in agreement with Wang [49]. This dissymmetry between nearby parallel fluid-driven fractures has also been observed by Haddad et al. [74] with a three-dimensional model for the simulation of plane vertical fractures generated from a horizontal well.

\subsubsection{Two opposed fluid-driven fractures}

The second test case focuses on the competition between two parallel fluid-driven fractures passing each other, as they extend from opposite directions. As previously, we search to assess the capability of the model to capture fracture propagation on non-planar directions. We consider a square bar of side $L=10 \mathrm{~m}$. The vertical displacements are blocked on the lower and upper edges and the horizontal displacements are blocked on the lateral edges. We introduce two vertical potential crack surfaces intersecting respectively the lower and upper edges of the square bar and distant of $d=2 m$ (see Figure 15 left). We inject the fluid with a constant flow rate $Q=3 \cdot 10^{-1} \mathrm{~kg} . \mathrm{s}^{-1}$ at both inlets for a total duration of $16 s$. The time step $\Delta t$ we use is $0.1 s$ during the first second and $0.25 s$ then (see once again section 4.4.4 for this choice).

The mesh used and the material characteristics are similar to the previous case. For the 6 first seconds, we do not update the potential crack surfaces so that both fluid-driven fractures propagate vertically, along the initially predefined path. Between $t=6 \mathrm{~s}$ and $t=16 \mathrm{~s}$, we update the potential crack surfaces each second.

The amplified deformed shape and the pore pressure obtained at $t=16 \mathrm{~s}$ are represented Figure 16 (right).
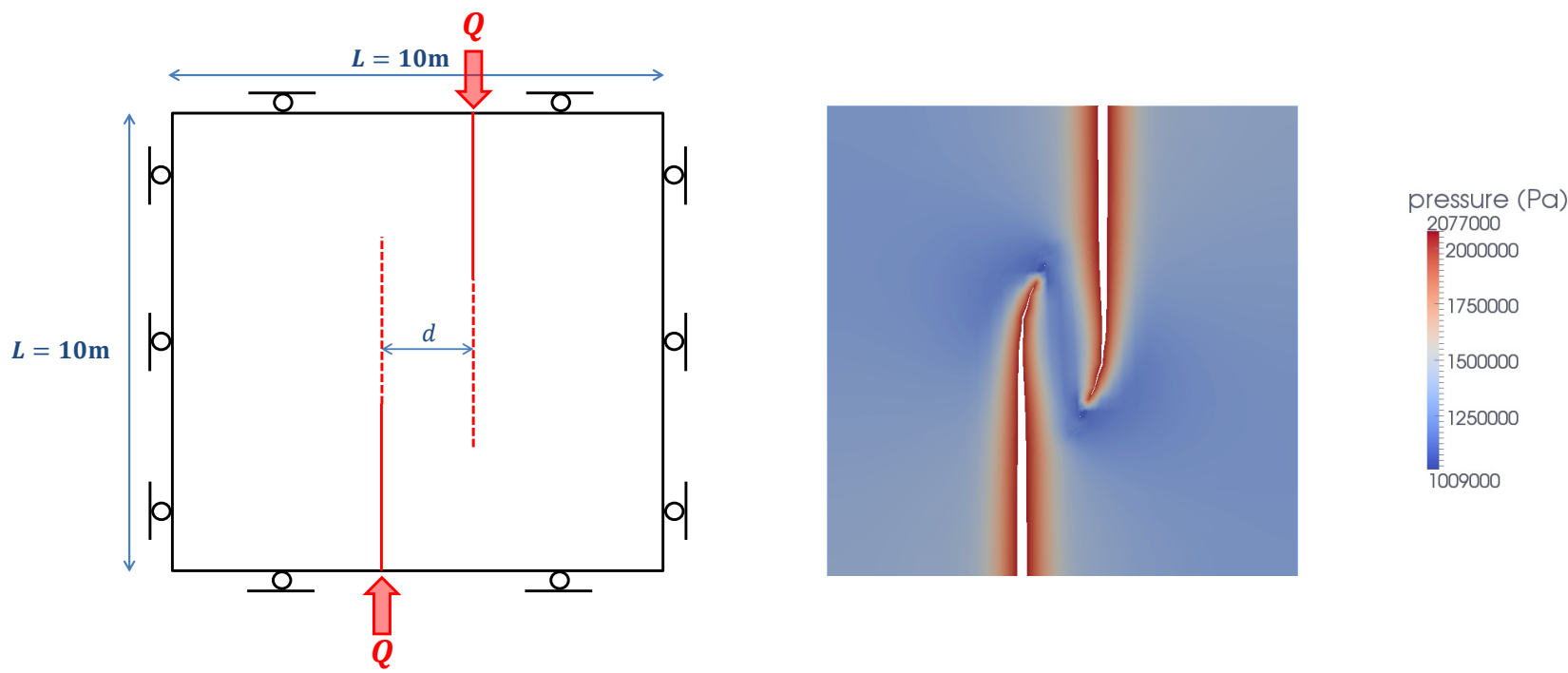

FIGURE 16: Two opposed fluid-driven fractures : geometry of the problem (left), amplified deformed shape (X100) and pore pressure at $t=16 \mathrm{~s}$ (right).

The two fluid-driven fractures propagate vertically during the first 6 seconds. Then, their trajectories fork progressively towards the inside of the domain, as if they were twisting around each other. At $t=16 \mathrm{~s}$, the angle between the fracture tips and the vertical plane is around $25^{\circ}$. In addition, the pattern we get is anti-symmetric with regards to the center of the domain, as the two fractures behave similarly.

Once again, the pattern we observe is similar to the one obtained by Wang [49] with an hydromechanical model coupled with XFEM, for a test case similar to the one we presented above. The bifurcation we observe is caused by a disturbance of the stress field in the vicinity of the fracture ends, due to the presence of the other fracture. This time, instead of pulling away, the two fluid-driven fractures roll over each other. 


\subsubsection{Reorientation of a $3 D$ fluid-driven fracture}

Finally, we perform a 3D reorientation test. We consider a block of height $H=6 m$, length $L=10 \mathrm{~m}$ and width $B=5 \mathrm{~m}$. The vertical displacements are blocked on the lower and upper faces and the displacements along the $y$ direction are blocked on the front and back faces. The block is submitted to a confining horizontal stress $\sigma_{0}=0.6 \mathrm{MPa}$ in the direction of $x$ (see Figure 17).

We introduce a planar potential crack surface, half-disc shaped, tilted with an angle $\theta=30^{\circ}$ compared to the horizontal plane (see Figure 177). The center of this potential crack surface coincides with the center of the front face of the block (in fact we model one half of a block containing a circular inclined fracture surface). We inject the fluid with a constant flow rate $Q=6 \mathrm{~kg} \cdot \mathrm{s}^{-1}$ at the center of the disc for $17 \mathrm{~s}$. The time step $\Delta t$ we use is $0.05 \mathrm{~s}$ during the first second and $0.25 \mathrm{~s}$ then, as explained next in section 4.4.4. The fluid fluxes are blocked on the front face.

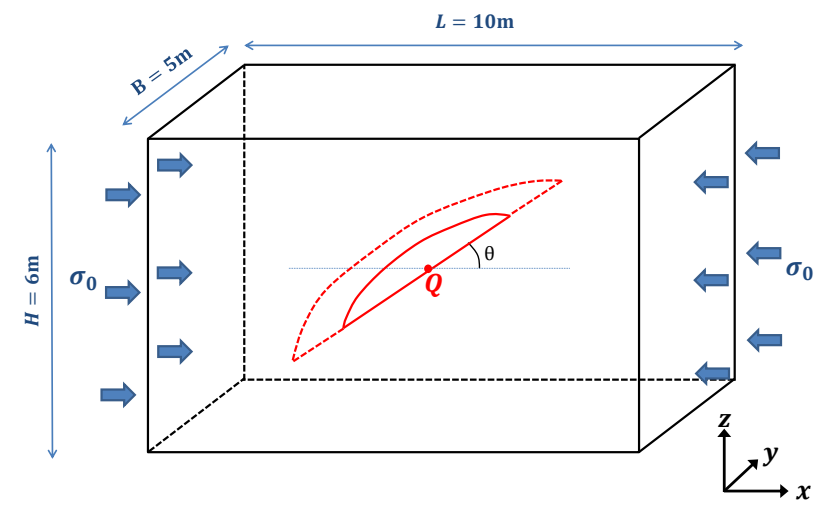

FIGURE 17: Geometry and loadings for the 3D fluid-driven fracture reorientation test.

The material and fluid characteristics are similar to the previous cases. The mesh we use is made of 22177 tetraedra. The fracture is forced to extend in the predefined potential direction until $t=2.5$ and extends on a non predefined path between $t=2.5 \mathrm{~s}$ and $t=17 \mathrm{~s}$. We proceed to the reorientation of the potential crack surface at $t=2.5 \mathrm{~s}, t=3 \mathrm{~s}$ and each second up to $t=17 \mathrm{~s}$.

The amplified deformed shape and the pore pressure obtained at $t=2.5 \mathrm{~s}$ and $t=17 \mathrm{~s}$ are represented Figure 18 (front view). At $t=2.5 \mathrm{~s}$, due to fluid injection, the fracture has extended over a half-disc which radius is approximately equal to $1.7 \mathrm{~m}$. Then, as expected, the fracture path gradually towards the direction of the maximum horizontal stress. As we can see in Figure 18. right), at $t=17 \mathrm{~s}$, the fluid-driven fracture has fully reoriented towards the horizontal plane, that is to say in the direction of the confining stress.
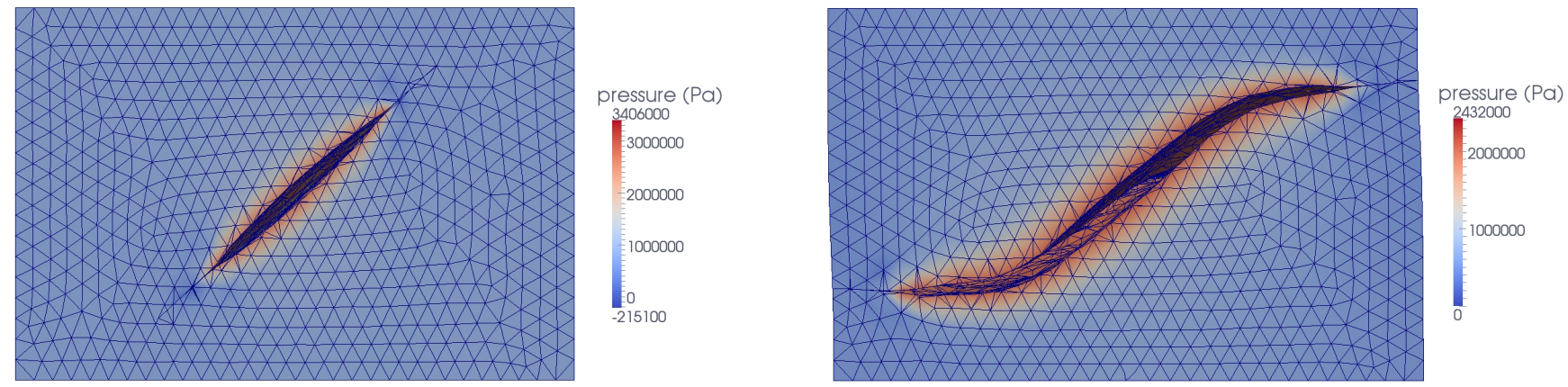

FIGURE 18: Amplified deformed shape (X1000) and pore pressure at $t=2.5 \mathrm{~s}$ (left) and $t=17 \mathrm{~s}$ (right) for the 3D fluid-driven fracture reorientation test. 
In order to better capture the fracture reorientation in the volume of the block, we represent the intersection points between the potential crack surface and the edges of the mesh. In Figure 19 (left), we represent the points of the initial potential crack surface and the pore pressure for $t=2.5 \mathrm{~s}$. We observe the propagation of the fluid-driven fracture on the red half disc (the red points correspond to a high fluid pressure). The transition zone between the red points (points that were reached by the hydraulic fracture) and the blue points (points that have not been reached by the hydraulic fracture) corresponds to the cohesive crack front at $t=2.5 \mathrm{~s}$. It is the limit between the adherent zone (in blue) and the debonding zone (in red). The first update of the potential crack surface is performed from this cohesive crack front. In Figure 19 (right), we represent the points of the initial potential crack surface and the pore pressure for $t=17 \mathrm{~s}$. The final potential crack surface was obtained from the initial potential crack surface after 15 successive updates. We observe a reorientation of the fracture surface towards the horizontal plane. At the crack front, the propagation performs in a direction close to the horizontal. The final potential crack surface is not perfectly anti-symmetric. It does not lead to a straight horizontal section on the back face of the block. This could be improved with the use of a finer mesh. We would then limit the irregularities in the computation of the stress intensity factors that are used to compute the bifurcation angle $\beta$ and obtain a smoother crack front and an entirely anti-symmetrical fracture surface.
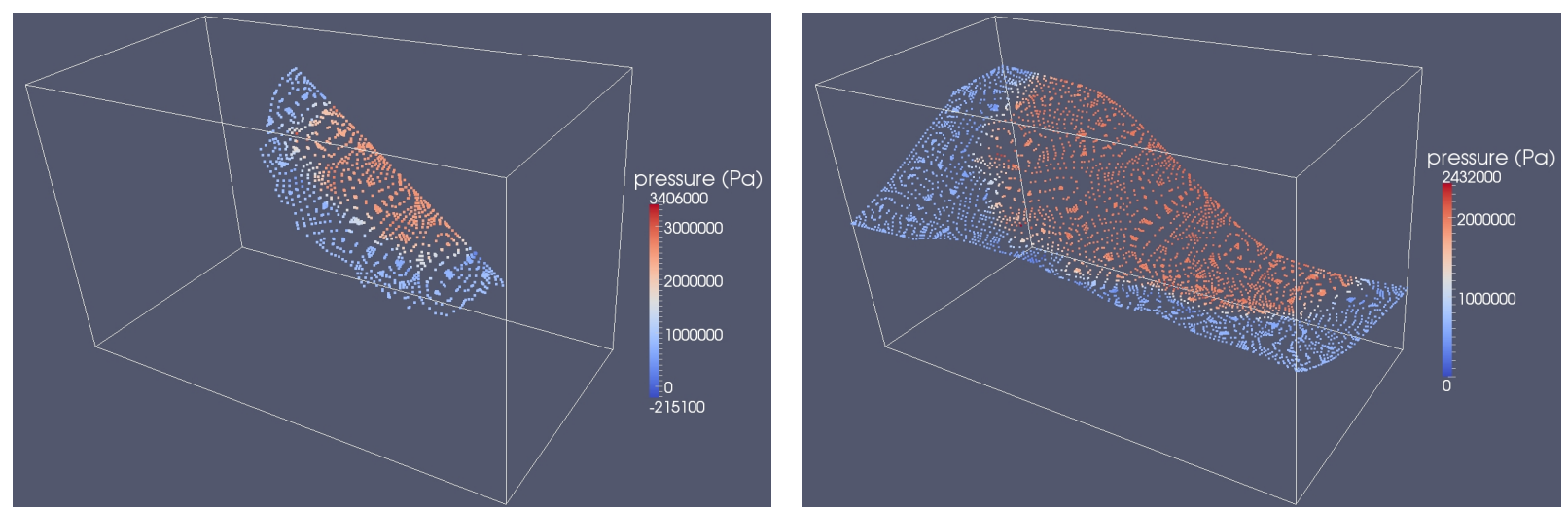

FIGURE 19: Points of the initial crack surface and pore pressure at $t=2.5 \mathrm{~s}$ (left) and points of the final crack surface and pore pressure at $t=17 \mathrm{~s}$ (right) for the 3D fluid-driven fracture reorientation test.

In Figure 20, we represent for two different view angles the initial potential crack surface and the pore pressure at $t=2.5 \mathrm{~s}$ (coloured points) and the final potential crack surface (white points). We can then observe the differences between the two potential crack surfaces. We verify that the deviation between the two surfaces takes place at the limit between between the debonding (in blue) zone and the cracked surface (in red) at $t=2.5 \mathrm{~s}$, that is to say the cohesive crack front at $t=2.5 \mathrm{~s}$.
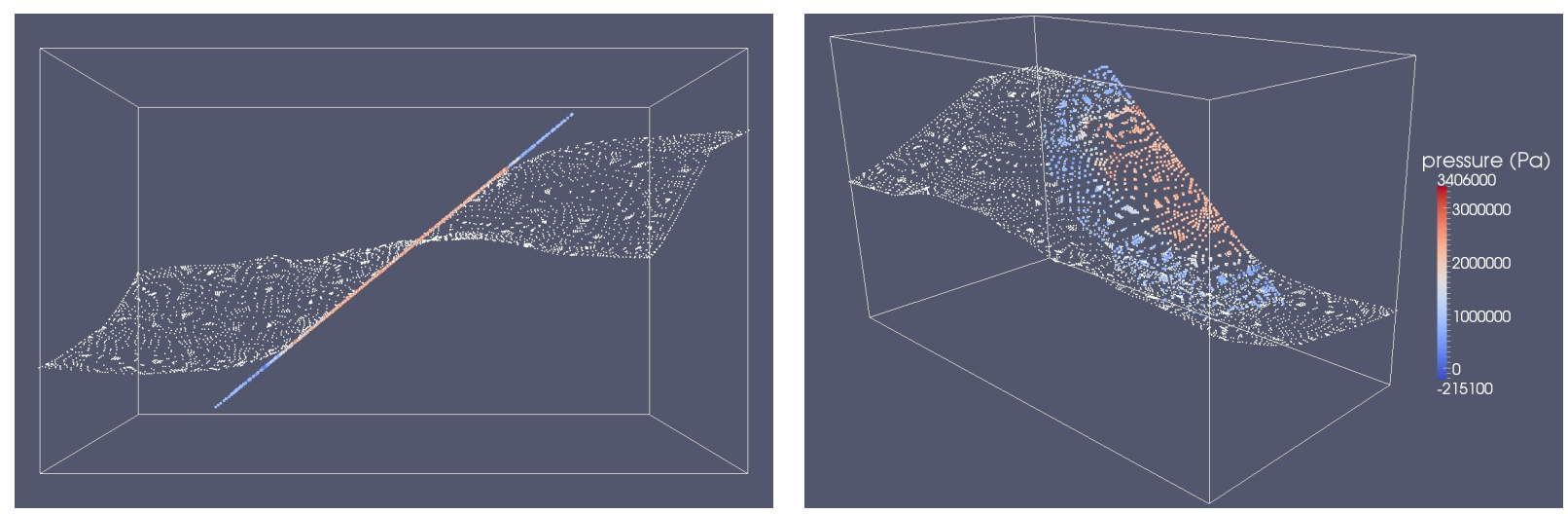

FIGURE 20: Points of the initial and final crack surfaces and pore pressure at $t=2,5 \mathrm{~s}$ for the 3D fluid-driven fracture reorientation test. 
This final test ends the validation procedure for the propagation of $3 \mathrm{D}$ cohesive fractures on non predefined paths. A physically consistent behavior, that is to say the reorientation towards the direction of the maximum confining stress, is observed and in agreement with the simulation carried out by Wang [48] in 2D. In addition, this test demonstrates the ability of our HM-XFEM model to handle complex 3D fracture surfaces.

\subsubsection{Remark}

In the 3 examples we presented in this chapter, the time step $\Delta t$ is $0.1 \mathrm{~s}$ or $0.05 \mathrm{~s}$ during the first second and $0.25 s$ then. The temporal discretization is calibrated to "accompany" the fracture propagation process, in accordance with mesh refinement. Indeed, with the injection flow rate we impose and the meshes we use, a time step of $0.25 \mathrm{~s}$ approximately corresponds to an extension over a length equivalent to half an element. We can then observe the dissipation of energy in the process zone and the propagation of the fluid-driven fractures. Regarding the update of the potential crack surface, the more frequently we perform the update, the more accurate the fracture path we obtain. We chose to update the potential crack surface each second. This frequency is a compromise between the computation costs and the accuracy of the obtained fracture path. During one second, the fluid-driven fractures extend over a distance approximately equivalent to the size of one or two elements. This frequency is then adapted to the refinement of the meshes we used.

\section{Extension of the model to fracture junctions}

\subsection{Junction level-sets}

In this section, we detail the extension of our model to fracture networks, with the introduction of junction elements. In order to model several distinct interfaces, we use several normal level-sets (see Figure 21 left), one for each interface. The approximation of the displacement field is then :

$$
\boldsymbol{u}_{h}(\boldsymbol{x})=\sum_{i \in N} \boldsymbol{a}_{i} \phi_{i}(\boldsymbol{x})+\sum_{n=1}^{\Pi} \sum_{j \in N \cap N_{H}^{n}} \boldsymbol{b}_{j}^{n} \phi_{j}(\boldsymbol{x}) H_{j}^{n}\left(l s n_{n}(\boldsymbol{x})\right)
$$

where $N$ is the set of nodes whose support contains the point $\boldsymbol{x}, N_{H}^{n}$ is the set of nodes that carry the Heaviside enrichment for the interface $n, \boldsymbol{b}_{j}^{n}$ are the associated additional degrees of freedom and $\Pi$ the number of distinct interfaces.
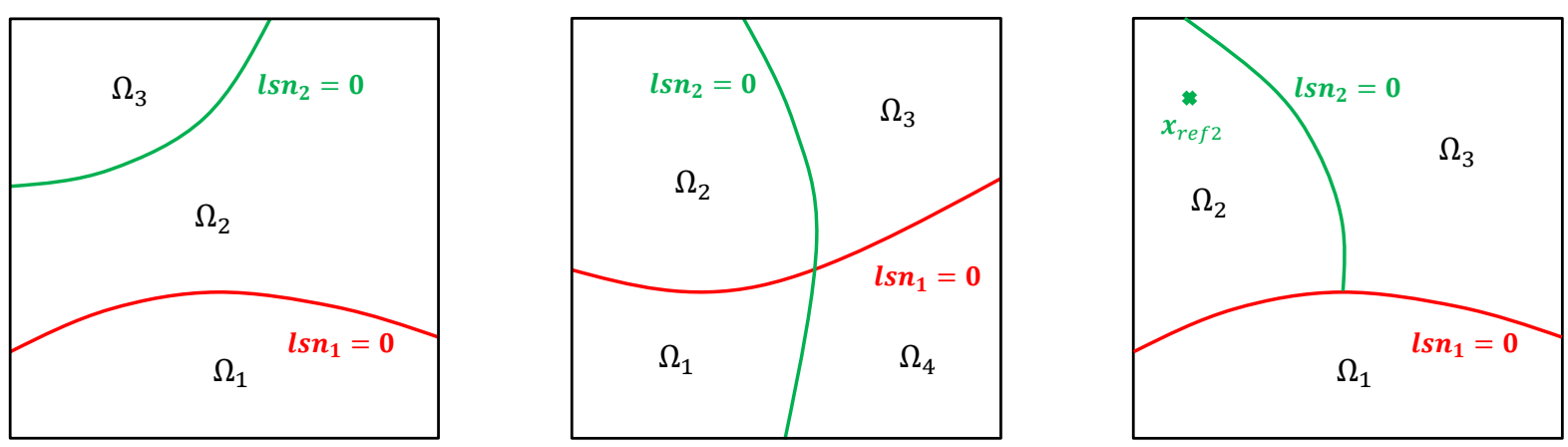

FIGURE 21: Two normal level-set fields defining two distinct interfaces (left), two crossing interfaces (middle) and two branched interfaces (right).

In contrast, once the interfaces are connected, we must apply a specific enrichment technique. Indeed, when two interfaces cross each other in a domain $\Omega$ (see Figure 21 middle), the domain $\Omega$ is partitioned into 4 subdomains $\Omega_{1}, \Omega_{2}, \Omega_{3}$ and $\Omega_{4}$. At the intersection, the kinematics of the four subdomains is described by : the set of classical degrees of freedom $\left(\boldsymbol{a}_{i}\right)$, the set of Heaviside degrees of freedom for the first interface $\left(\boldsymbol{b}_{j}^{1}\right)$ and the set of Heaviside degrees of freedom for the second interface $\left(\boldsymbol{b}_{j}^{2}\right)$. However, 4 sets of displacement degrees of freedom are necessary to properly describe the kinematics of the 4 subdomains $\Omega_{1}, \Omega_{2}, \Omega_{3}$ and $\Omega_{4}$ at the intersection (to each new subdomain is associated a new set of displacement degrees of freedom). Consequently, we make the choice suggested in [75] which 
consists in branching secondary interfaces on main interfaces. In Figure 21(right), the secondary interface (in green) is branched on the upper wall of the main interface (in red). At the junction, the kinematics of the 3 subdomains $\Omega_{1}, \Omega_{2}$ and $\Omega_{3}$ is properly described by the set of classical degrees of freedom and the two sets of enriched degrees of freedoms associated to the two interfaces. In order to model an intersection, we could branch another secondary interface on the other side of the main interface. We would then dispose of four sets of degrees of freedom to model the kinematics of the intersection. In practice, in order to define on which side of the main interface the branching is performed, the user is invited to advise a reference point $\boldsymbol{x}_{\text {ref }}$ located on the side corresponding to the branching. In Figure 21 (right), the point $\boldsymbol{x}_{r e f 2}$ is located above the main interface. The secondary interface is then branched on the upper wall of the main interface. This hierarchical procedure can be extended to successive branching. Thus, an interface can be branched on a secondary interface that is itself branched on a first interface (see Figure 22 left).
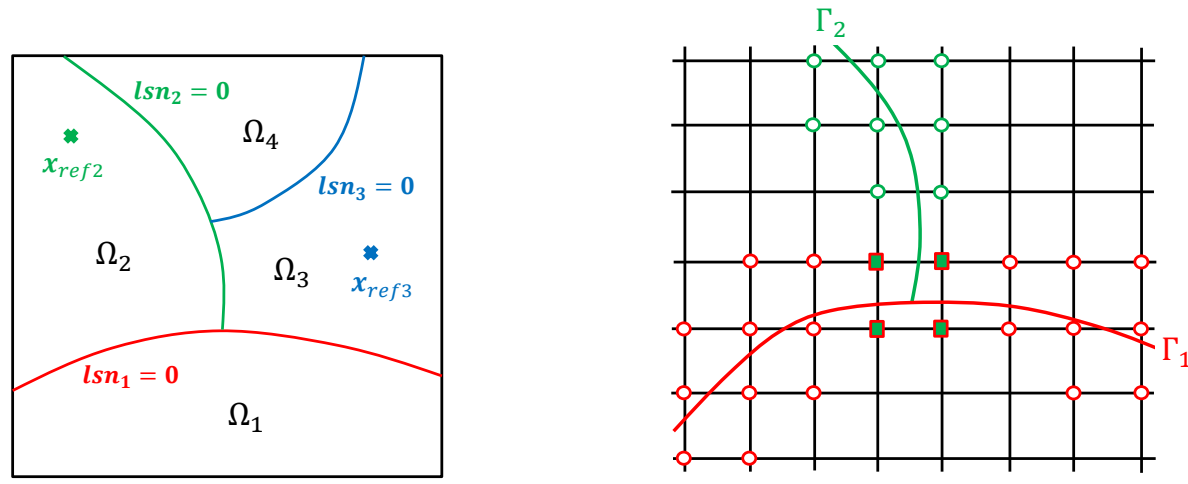
O: node enriched for the interface $\Gamma_{1}$ O: node enriched for the interface $\Gamma_{2}$ : node enriched for the interface $\Gamma_{1}$ and for the junction of $\Gamma_{2}$ on $\Gamma_{1}$

FIGURE 22: Successively branched interfaces (left) and Heaviside enrichment for an interface junction (right).

\subsection{Discretization with XFEM}

\subsubsection{Fields related to the bulk}

Regarding the approximation of the displacement field, we still use the Heaviside enrichment presented in paragraph 2.4.1 for the main interface. But for the secondary interface, we must truncate this enrichment with respect to the junction. Indeed, a secondary interface is defined only on one side of its associated main interface. For this aim, we adopt the approach introduced by Daux et al. [75]. The truncation is obtained by a specific junction enrichment function $J^{2}$ for the secondary interface (see Figure 22 right where secondary interface $\Gamma_{2}$ is branched on the main interface $\Gamma_{1}$ ). The junction function $J^{2}$ corresponds to the Heaviside function for the branched interface $\Gamma_{2}$ (similarly to what was presented in paragraph 2.4.1 but it is truncated with respect to the junction. It is prolonged with a zero value on the side where $\Gamma_{2}$ is not defined :

$$
J_{j}^{2}(\boldsymbol{x})=\left\{\begin{array}{cl}
H_{j}^{2}\left(l s n_{2}(\boldsymbol{x})\right) & \text { if } l s n_{1}(\boldsymbol{x}) * l s n_{1}\left(\boldsymbol{x}_{r e f 2}\right) \geq 0 \\
0 & \text { otherwise }
\end{array}\right.
$$

In practice, the junction enrichment is used only when necessary. For a secondary interface $\Gamma_{2}$ branched on a main interface $\Gamma_{1}$, only the nodes whose support contains the junction are concerned by the junction enrichment. The other nodes whose support is fully intersected by the interface $\Gamma_{2}$ carry the generic Heaviside enrichment for the interface $\Gamma_{2}$ (see Figure 22 right). The approximation of the displacement field is then :

$$
\boldsymbol{u}_{h}(\boldsymbol{x})=\sum_{i \in N} \boldsymbol{a}_{i} \phi_{i}(\boldsymbol{x})+\sum_{j \in N \cap N_{H}^{1}} \boldsymbol{b}_{j}^{1} \phi_{j}(\boldsymbol{x}) H_{j}^{1}\left(l s n_{1}(\boldsymbol{x})\right)+\sum_{k \in N \cap J_{2 \rightarrow 1}} \boldsymbol{b}_{k}^{2} \phi_{k}(\boldsymbol{x}) J_{k}^{2}\left(l s n_{2}(\boldsymbol{x})\right)+\sum_{j \in N \cap N_{H}^{2}} \boldsymbol{b}_{j}^{2} \phi_{j}(\boldsymbol{x}) H_{j}^{2}\left(l s n_{2}(\boldsymbol{x})\right)
$$

where $N_{H}^{1}$ is the set of nodes whose support is fully intersected by the interface $\Gamma_{1}, N_{H}^{2}$ the set of nodes whose support is fully intersected by the interface $\Gamma_{2}$ and $J_{2 \rightarrow 1}$ the set of nodes whose support contains the branching of $\Gamma_{2}$ on $\Gamma_{1}$. 
The junction enrichment function can be extended to $n$ successive branchings from a main interface $\Gamma_{1}$. The junction function $J^{n}$ associated to the last interface $\Gamma_{n}$ (at the end-of-chain) is then :

$$
J_{j}^{n}(\boldsymbol{x})=\left\{\begin{array}{cl}
H_{j}^{n}\left(l s n_{n}(\boldsymbol{x})\right) & \text { if } \forall i \in\{1,2, \ldots, n-1\}, l s n_{i}(\boldsymbol{x}) * l s n_{i}\left(\boldsymbol{x}_{r e f n}\right) \geq 0 \\
0 & \text { otherwise }
\end{array}\right.
$$

The additional sets of degrees of freedom are added incrementally, as the interfaces are being introduced. For each node, there is a correspondance table that associates the set of Heaviside degrees of freedom $\boldsymbol{b}_{j}^{n}$ to the interfaces that intersect the support of the node. In the code we use, the nodes of the mesh can not carry more than 4 sets of additional degrees of freedom. Consequently, the support of a node can not be intersected by more than 4 interfaces. When this condition is violated, the code returns an error message inviting the user whether to use a finer mesh or to decrease the number of interfaces in the model. Therefore the number of interfaces our HM-XFEM model can handle is limited but could be enriched on demand without difficulty, if necessary.

Another approach for branched discontinuities has been proposed by Simone et al. [76] in the context of GFEM [77]. It suggests to use the subdomain characteristic functions to build the enrichment. To each subdomain is then associated a set of degrees of freedom. This approach seems easier and more practical to implement but the treatment of crack fronts is problematical as it only involves closed contours. Duarte [78] generalized this approach for 3D fractures by extending the fractures to the domain boundaries, but this requires a significant refinement in the crack tip area. In the approach we adopted, which was introduced by Daux et al. [75], the treatment of the junctions relies on a local enrichment strategy and the additional degrees of freedom are associated to the discontinuities, not the subdomains. Then nothing prevents from considering cracks instead of interfaces, provided that the crack tips are sufficiently distant from the junction, in order to ensure that the nodes whose support contains the junction are entirely cut by the different fracture branches of the junction (see Figure 22,

\subsubsection{Fields related to the fracture}

We aim at simulating fluid-driven fracture networks within porous media. It is then necessary to propose a framework for the simulation of fluid-driven fracture junctions. In paragraph 5.2.1, we have detailed the spatial discretization for the approximation of the displacement field in presence of fracture junctions. The same discretization applies for the pore pressure field, except that it is linearly interpolated. The discretization of the fields associated to the cohesive fractures $\left(p_{f}, q_{+}, q_{-}, \lambda, \mu, \boldsymbol{w}\right)$ is more complicated and we need to introduce appropriate approximation spaces, in order to account for the cohesive stresses as well as for the fluid flow at the fracture junctions. In particular, we must account for the cohesive stresses in each branch of a fracture junction and hydraulically connect the different fracture branches.

In the literature, there are few examples for the simulation of contact or cohesive stresses in fracture junction areas. The work of Sanders [79] accounts for the contact along branched discontinuities with the GFEM approach introduced by Simone et al. [76] for 2D polycristals. In the context of XFEM, Siavelis [80, 59] proposed an augmented Lagrangian model for contact under friction and large sliding along branched interfaces. The work of Siavelis constitutes a basis for the extension of our HM-XFEM model to fluid-driven fracture junctions.

The modeling of fracture junctions within porous media is more commonly encountered. The hydromechanical model developed by Pouya [81] accounts for fluid-driven fracture junctions with interface elements. It is notably suited for the measurement of the hydraulic properties of porous fractured media. As a matter of fact, the presence of connected fluid-filled interfaces in a porous medium induces a significant increase in its equivalent permeability [82]. However, this model is limited to bi-dimensional simulations and requires to generate a mesh conforming to the fracture network.

In our model, for a single cohesive interface, the construction of the approximation space for the cohesive fields has been depicted in paragraph 2.4.2 It relies on the vertex nodes of the edges intersected by the interface. Equality relations are enforced on both sides of the interface, in order to reduce the approximation space and satisfy the LBB stability condition [23]. When the model contains several non connected interfaces, a proper approximation space is associated to each interface. This way, when two interfaces intersect the same element, we must dispose of two sets of degrees of freedom for the fields associated to the cohesive interfaces.

In contrast, in presence of a junction between two interfaces, the second one being branched on the first one, we consider not 2 but 3 distinct interfaces for the construction of the approximation spaces associated to the cohesive 
interfaces. Indeed, one interface being branched on a second interface generates 3 branches (see Figure 23). These branches need to be treated separately to ensure the relevance of the approximation spaces with respect to the junction. The main interface is then divided into two distinct branches.
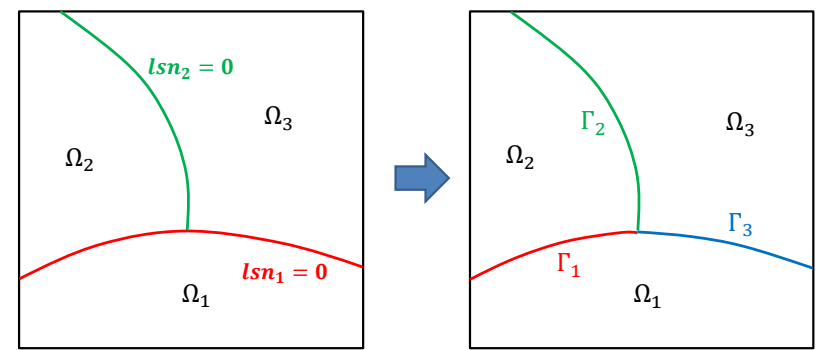

FIGURE 23: An interface junction defined by two normal level-sets (left) and the three resulting interface branches (right).

In Figure 24(left), we represent the approximation spaces for the fields associated to the cohesive interfaces in presence of a junction. For an interface $\Gamma_{i}$, the approximation space is based on a set of active nodes : the vertex nodes of the edges intersected by the interface $\Gamma_{i}$. Equality relations are imposed between the vertex nodes of the dotted edges. The vertex nodes linked by dotted edges then carry the same degree of freedom $\Lambda_{i}$ for the cohesive field $\Lambda$. We systematically consider as many interfaces as branches in presence of junctions for the construction of the approximation spaces associated to the cohesive interfaces because the cohesive fields in each branch are $a$ priori independent and must be discretized over distinct approximation spaces. For instance, the contact pressure is independent from one branch to the other. Furthermore, in the context of fluid-driven fracture junctions, omitting to treat the main interface as two distinct branches may skew the bifurcation phenomenon we expect to observe at the junctions (due to the continuity of the cohesive fields for the branches resulting from the main interface).

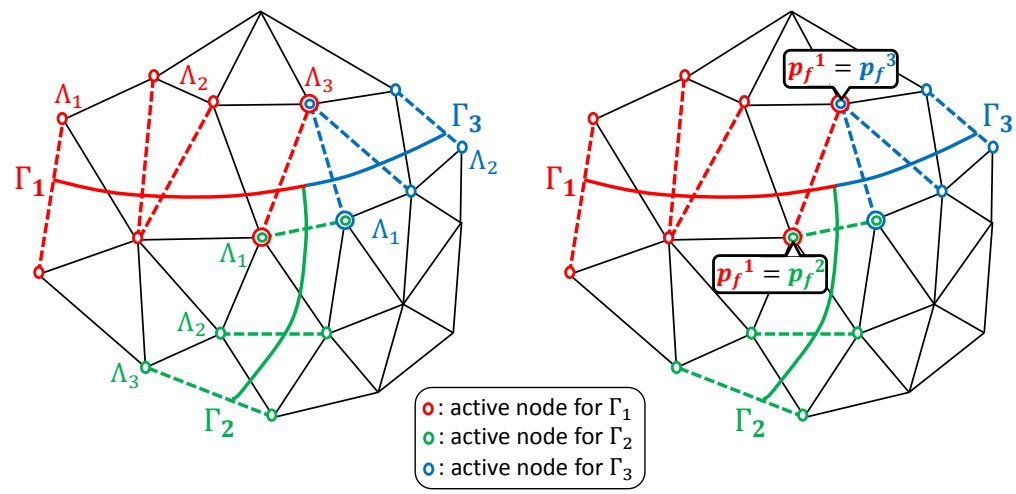

FIGURE 24: Approximation spaces for the fracture branches at a junction (left) and continuity of the fluid pressure at the junction (right).

In the element that contains the interface junction, 3 sets of degrees of freedom are then necessary for the fields associated to the cohesive interfaces. In Figure 25, we represent a quadrangle element containing an interface junction and its degrees of freedom. Similarly to what is done for the displacements and pore pressure fields, the sets of degrees of freedom for the fields associated to the cohesive interfaces are added sequentially as the interfaces are introduced in the model. For each node, we define a correspondence table that associates the cohesive degrees of freedom with the different interfaces intersecting the support of the node. 


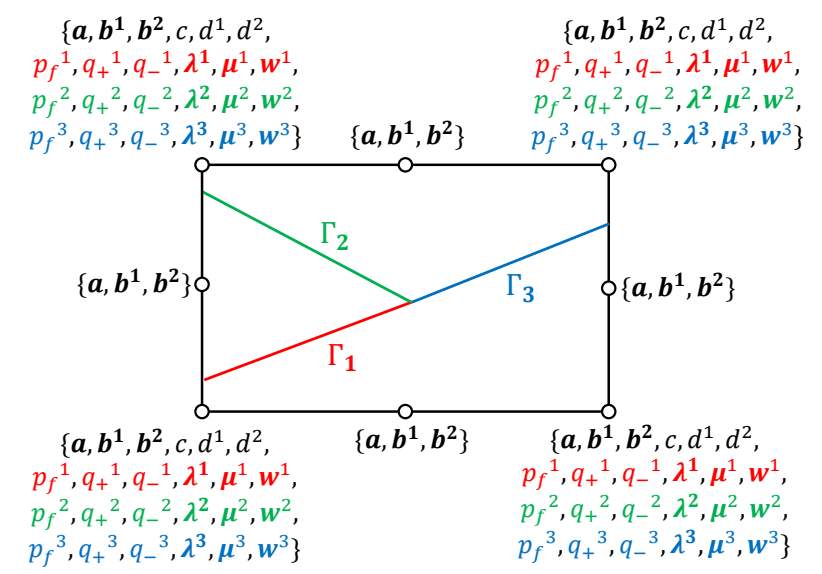

FIGURE 25: A quadrangle cohesive HM-XFEM element containing a junction.

\subsection{Mass exchanges at the junction}

When it comes to hydraulic fracture junctions, in order to ensure the "hydraulic connection" between each branch, we impose the continuity of the fluid pressure $p_{f}$ from one branch to another. In practice, we impose this condition directly at the vertex nodes of the elements containing a junction. In Figure 24 (right), the fluid pressure $p_{f}$ is constant in each branch in the element containing the junction. We enforce the condition $p_{f}^{1}=p_{f}^{2}$ on the vertex node active for both $\Gamma_{1}$ and $\Gamma_{2}$ and $p_{f}^{1}=p_{f}^{3}$ on the vertex node active for both $\Gamma_{1}$ and $\Gamma_{3}$. In the end, we have enforced $p_{f}^{1}=p_{f}^{2}=p_{f}^{3}$. The fluid pressure $p_{f}$ is then constant in the element containing the junction. We do not enforce the condition $p_{f}^{2}=p_{f}^{3}$ on the vertex node active for both $\Gamma_{2}$ and $\Gamma_{3}$ in order to prevent a redundancy. The procedure to enforce the continuity of the fluid pressure $p_{f}$ at the junctions of interfaces follows the steps below :

- for all the elements containing a junction, we enforce equality relations between the degrees of freedom $p_{f}$ on the vertex nodes active for at least two distinct branches,

- then, within each element, we remove the redundant equality relations,

- finally, we compare the equality relations enforced from one element to the adjacent elements in order to once again remove redundant relations.

\subsection{Multi-stage hydraulic fracturing}

In order to illustrate the abilities of our HM-XFEM model extended to discontinuity junctions, we proceed to "multi-stage hydraulic fracturing" tests. This process consists in injecting pressurized water in horizontal wells in order to propagate prior initialized vertical fractures. Besides the orientation of the well, that must be insightfully chosen depending on the regional stress state, the key parameter for the success of this technique is the spacing between the pre-initialized vertical fractures. If this spacing is taken too small, we may observe the propagation of a reduced number of vertical fractures, because of the stress shadowing effect [83], [84]. Indeed, when a vertical fluid-driven fracture propagates, it modifies the stress state in its neighborhood. It induces significant stresses in the horizontal direction, making it more difficult for other vertical hydraulic fractures to propagate.

Recent works focus on the propagation of multiple vertical fluid-driven fractures, powered by the same horizontal well [85], [86]. In particular, Bunger [85] evaluates the minimum fluid flux to inject in the horizontal well to propagate simultaneously the vertical fractures. An hydrodynamic template handles the partition of the fluid flux in the different vertical fractures, connected via the horizontal well. Thus, the hydraulic junctions between the fractures and the horizontal well are simulated with a balanced fluid partitioning algorithm. Lecampion studied in details the competition between vertical fluid-driven fractures in the context of "multi-stage hydraulic fracturing" [86] in light of a dimensionless number that depends on the injection rate, the rock toughness and the spacing between the fractures. This dimensionless number governs the transition between a regime in which all fractures grow equally to the regime of preferential growth of the outer fractures in the array. 
We propose to study this problem with our HM-XFEM model. We consider a block of height $H=10 \mathrm{~m}$ and width $L=120 \mathrm{~m}$ crossed in its lower part by a horizontal interface (see Figure 26). Several vertical interfaces, initially closed, are branched on the upper wall of the horizontal interface. The intersections between the horizontal well and the vertical fractures are then handled with junction elements, ensuring that mass conservation for the fluid is satisfied in the whole system. The fractures are supposed vertical and the propagation direction is assumed to be known $a$ priori so that we do not proceed to updates of the fracture paths. On the upper edge of the domain, we set to 0 the Heaviside degrees of freedom associated to the horizontal displacement jump, in order to prevent the opening of the vertical interfaces on this edge. The spacing between two vertical interfaces $2 l$ is taken twice as large as the spacing between the lateral edges and the vertical interfaces facing these edges $l$, in order to generate a periodic pattern with a $2 l$ period and to limit the side-effects.

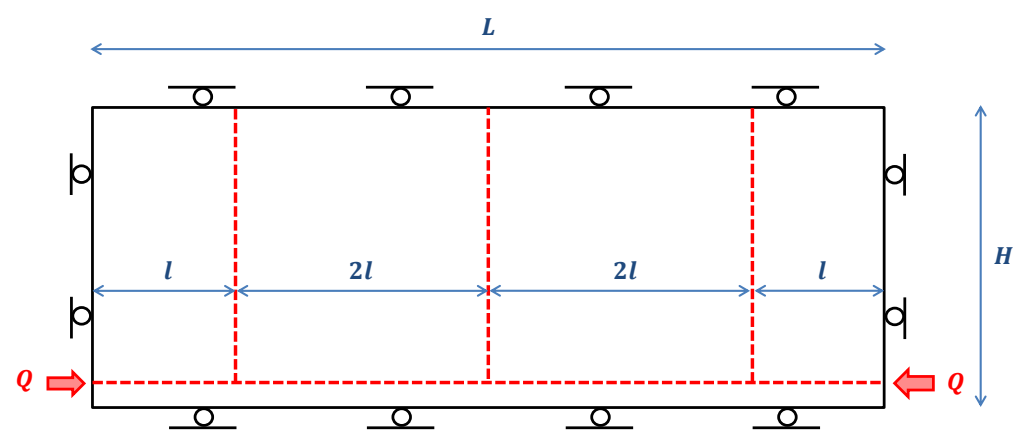

FIGURE 26: Geometry for the simulation of "multi-stage hydraulic fracturing".

The horizontal interface acts as the horizontal well. It is initially open. It is powered with fluid at both ends, and the fluid pressure homogenizes very rapidly in this interface. The initial amplified deformed shape and the initial pore pressure are represented in Figure 27. The fluid pressure in the well is then in the order of 6.7MPa. We carry on the injection of fluid with a constant flow rate $Q=1 \cdot 10^{-1} \mathrm{~kg} . \mathrm{s}^{-1}$ for $1500 \mathrm{~s}$.

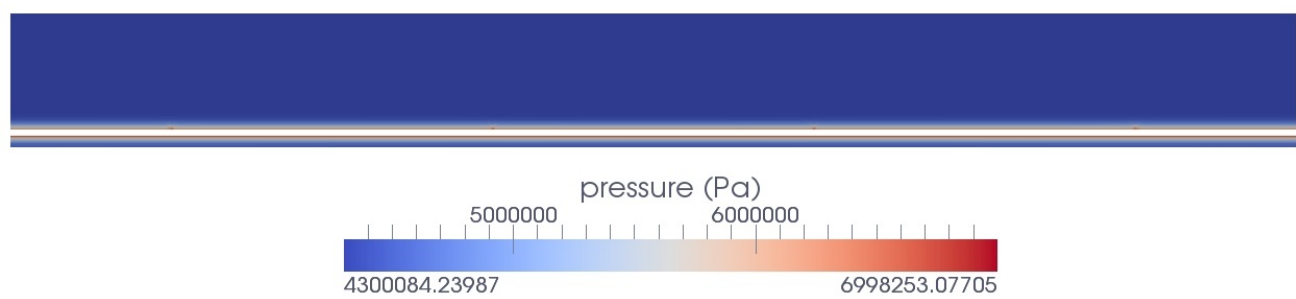

FIGURE 27: Initial state for the "multi-stage hydraulic fracturing" simulations.

The parameters for the matrix and the fluid are summarized in Table 3 


\begin{tabular}{|l|l|}
\hline Young's modulus & $E=5800 \mathrm{MPa}$ \\
Poisson's ratio & $v=0.25$ \\
Density of the fluid & $\rho=1000 \mathrm{~kg}^{-\mathrm{m}^{-3}}$ \\
Biot's coefficient & $b=0.8$ \\
Intrinsic permeability & $k^{\text {int }}=10^{-17} \mathrm{~m}^{2}$ \\
Viscosity of the fluid & $\mu=0.001 \mathrm{~Pa} . \mathrm{s}$ \\
Porosity & $\phi=0.1$ \\
Compressibility modulus of the fluid & $\frac{1}{K_{w}}=5 \cdot 10^{-10} \mathrm{~Pa}^{-1}$ \\
Critical stress & $\sigma_{c}=0.9 \mathrm{MPa}$ \\
Cohesive energy & $G_{c}=500 \mathrm{~Pa} . \mathrm{m}$ \\
Augmentation parameters & $r=r_{s}=2$ \\
\hline
\end{tabular}

TABLE 3: Parameters for the porous medium and the fluid

We use a regular mesh made of 10860 quadrangles. Simulations are conducted for two different values of fracture spacing, ie. with an initial number $n$ of vertical discontinuities equal to 4 and 5, respectively. The amplified deformed shapes and the pore pressure fields obtained at $t=1500 \mathrm{~s}$ are represented in Figure 28 for both configurations.
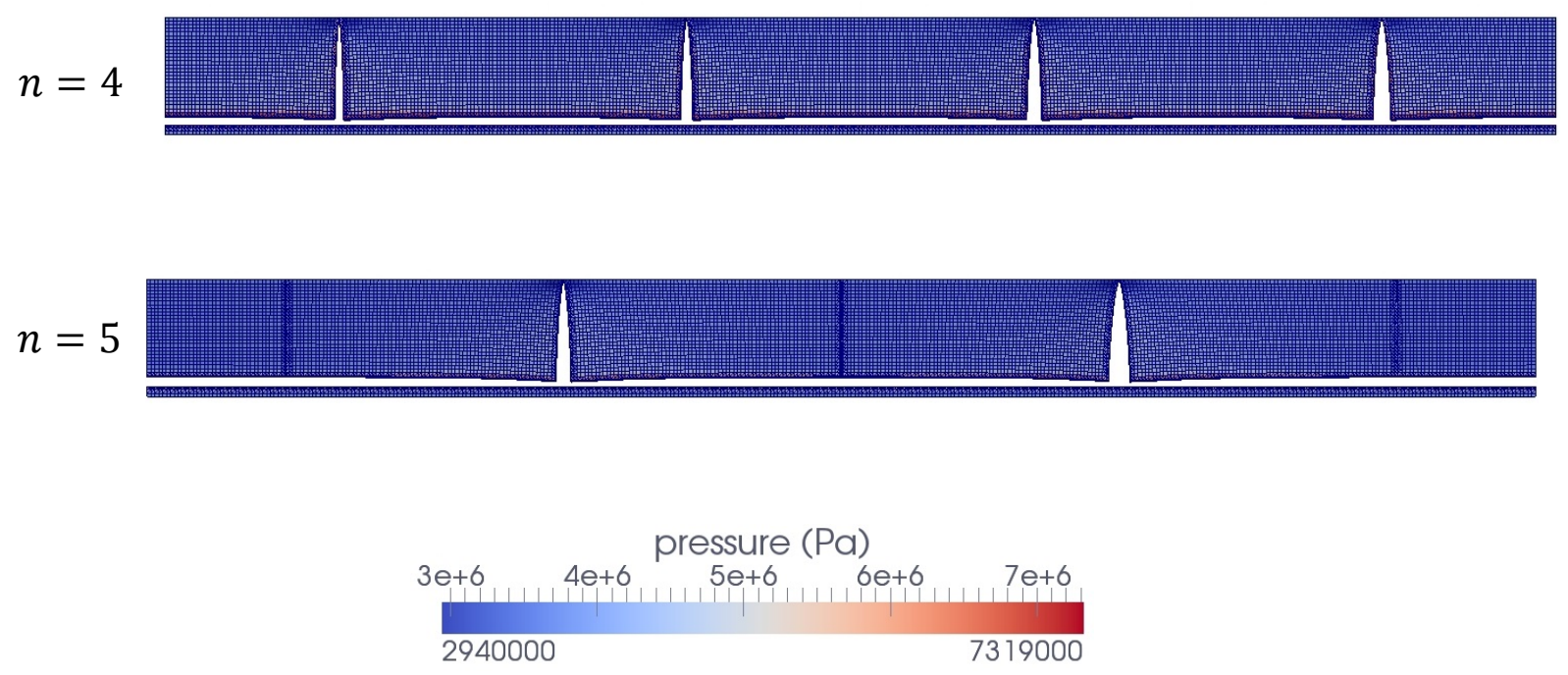

FIGURE 28: Amplified deformed shapes (X100) and pore pressure field for the first series of "multi-stage hydraulic fracturing" simulations ( $2 l=24 \mathrm{~m}$ for $n=5,2 l=30 m$ for $n=4)$.

We observe the propagation of some of the vertical interfaces. For both cases, these propagations are quasisimultaneous and very brutal. It takes approximately $1 s$ to fully propagate a vertical interface. When $n=4$, all the vertical fractures propagate. No mutual interaction between adjacent fractures is observed. The spacing is then $2 l=30 \mathrm{~m}$. In contrast, for $n=5$ and a spacing $2 l=24 m$, only one interface out of two propagates. A highly compressive zone occurs in the inter-fractured region, classically referred to as the stress shadowing effect, promoting preferential growth of some discontinuities. A direct conclusion is that the minimal spacing for the propagation of all the vertical interfaces with no interferences is located between $24 m$ and $30 m$ (for this specific case). The choice we made to model the well as an interface ensures that the mass balance for the fluid in the system \{well+fractures\} is solved within the finite elements. It is enabled by our HM-XFEM junction elements. A careful analysis of patterns in Figure 28, however, reveals that fractures are not perfectly symmetric with respect to the vertical axis at $x=L / 2$. Small differences in the fracture apertures are visible. Indeed, the subdivision of mesh elements cut or adjacent to the discontinuities as required by the discretization with the XFEM-method (see [34] for more details) results in a non-uniform mesh that breaks the symmetry of our simulations. Minor changes in the mesh subdivision procedure is expected to palliate this deficiency. 
To go further, we proceed to a second series of tests but with a constant number $n$ of fractures, in order to refine the range for the minimum spacing allowing all the vertical fractures to propagate. We make vary the spacing $2 l$ between $24 \mathrm{~m}$ and $30 \mathrm{~m}$ with a stepsize of $1 \mathrm{~m}$ (data not shown). The number of vertical interfaces being equal to 4 , the width of the domain is taken equal to $4 * 2 l$. For $2 l \geq 25 m$, we systematically observe the propagation of all the vertical interfaces. For $2 l=24 m$, only the extremal vertical interfaces propagate. This second series of tests reinforces the assumption of the existence of a minimum spacing for the simultaneous propagation of the whole set of vertical interfaces. In this specific case, we infer that the minimum spacing is between $24 \mathrm{~m}$ and $25 \mathrm{~m}$. Nonetheless, a thorough analysis should be conducted to consider with certainty the validity of this result.

These two series of tests illustrate the capabilities of our HM-XFEM model with junctions, which may constitute a powerful numerical tool for various industrial applications.

\section{Conclusion}

We have developed in the present work a 3D fully coupled numerical model to simulate fluid-driven propagation of multiple fractures in porous medium. The choice was made to use the XFEM in order to circumvent the difficulty to generate meshes conforming the fracture networks. The stability of this strongly coupled model relies on three distinct approximation spaces. Advanced numerical methods have been used to overcome the well-known weaknesses of 3D quadratic XFEM elements that suffer classically from major robustness and conditioning issues. First, a robust procedure has been developed in [57] for the integration of functions discontinuous across arbitrary curved interfaces. It is combined with an improved XFEM enrichment technique proposed by [56] and well-suited for strong discontinuities. Second, a novel mortar formulation derived from [58] is employed for the cohesive zone model.

The HM-XFEM model has shown promising results on the KGD benchmark [34] as well as on the penny-shaped benchmark. Then, various reorientation tests have demonstrated the ability of our model to handle the propagation of fluid-driven fractures on non-predefined paths, involving complex fracture geometries. Finally, we exposed the extension of our model to fracture junctions and illustrated, with series of "multi-stage hydraulic fracturing" simulations, the range of applications offered by this additional feature.

As a conclusion, significant extensions to the HM-XFEM model initially developed by Faivre et al. [34] have been introduced and the resulting model has demonstrated powerful capabilities to capture crack dynamics with particular emphasis on the prediction of complex 3D fracture path. As far as we know, this is the very first attempt to derive an XFEM based-model including all these features. In spite of these advances, limitations remain that will need further developments. Up to now, the model is only able to account for a limited number of fractures and is therefore adapted to simulate only major fractures. The secondary fracture network could be considered in the model by means of homogenization techniques. The nucleation of cracks in porous media as well as fracture bifurcation is not treated either in our model. We model the evolution of pre-existing fractures and the eventual fracture junctions are initially set by the user. A method that enables the simulation of crack nucleation and coalescence in the context of XFEM and Thick Level-Set approach was recently developed by Moës et al. [87] and could be implemented. An automatic procedure for the detection of new junctions after updating fractured paths will also be developed.

\section{Acknowledgments}

This work was financially supported by EDF and IFPEN that we thank for their participation. This research was supported in part by the French National Research Agency through the national program Investissements davenir with the reference ANR-10-LABEX-21-RESSOURCES21 and by the Regional Council of Lorraine. Also, this research has received financial support from the French National Research Agency (ANR) through the HydroGeoDam project under grant no. ANR-17-CE06-0016.

\section{References}

[1] M. Zoback, S. Gorelick, Earthquake triggering and large-scale geologic storage of carbon dioxide, PNAS 109 (2012) 10164-10168.

[2] R. Nordgren, Propagation of a vertical hydraulic fracture, J. Geophys. Res. 12 (1972) 306-314.

[3] T. Mura, H. Abe, L. Keer, Growth-rate of a penny-shaped crack in hydraulic fracturing of rocks, J. Geophys. Res. 81 (1976) 35-40. 
[4] A. Savitski, E. Detournay, Propagation of a penny-shaped fluid-driven fracture in an impermeable rock : asymptotic solutions, International Journal of Solids and Structures 39 (2002) 6311-6337.

[5] Z. Chen, A. Bunger, X. Zhang, R. Jeffrey, Cohesive zone finite element-based modeling of hydraulic fractures, Acta Mech. Solida Sinica 22 (2009) 443-452.

[6] B. Carrier, S. Granet, Numerical modeling of hydraulic fracture problem in permeable medium using cohesive zone model, Engineering Fracture Mechanics 79 (2012) 312-328.

[7] T. K. Perkins, L. R. Kern, Widths of fractures, J. Pet. Technol. 222 (1961) 937-949.

[8] S. Khristianovic, Y. Zheltov, Formation of vertical fractures by means of highly viscous fluids, Proceedings of the 4th world petroleum congress 2 (1955) 579-586.

[9] I. D. Palmer, H. B. Carroll, Three-dimensional hydraulic fracture propagation in the presence of stress variations, Proceedings of the SPE/DOE/GRI unconventional gas recovery symposium, SPE/DOE 10849 (1982) 870-878.

[10] J. Shao, Poroelastic behaviour of brittle rock materials with anisotropic damage, Mech. Mater. 30 (1998) 41-53.

[11] J. Shao, Y. Lu, D. Lydzba, Damage modeling of saturated rocks in drained and undrained conditions, J. Eng. Mech. ASCE 130 (2004) 733-740.

[12] S. Andrieux, Y. Bamberger, J. Marigo, Un modèle de matériau microfissuré pour les roches et les bétons, Journal de Mécanique théorique et appliquée 5 (1986) 471-513.

[13] J. Noorishad, C.F.Tsang, P. Witherspoon, Theoretical and field studies of coupled hydromechanical behaviour of fractured rocks -1. development and verification of a numerical simulator, International Journal of Rock Mechanics and Mining Sciences \& Geomechanics Abstracts 29 (1992) 401-409.

[14] A. Cornec, I. Scheider, K.H.Schwalbe, On the practical application of the cohesive model, Engng. Fract. Mech. 70 (1963) $1963-1987$.

[15] P.A.Witherspoon, J.S.Y.Wang, K. Iwai, J.E.Gale, Validity of the cubic law for fluid flow in a deformable rock fracture, Water Resour. Res. 16 (1980) 1016-1024.

[16] D. Owen, M. Lobao, R. Eve, N. de Souza, Modeling of hydro-fracture flow in porous media, Engng. Comput. 27 (2010) 129-154.

[17] D.I.Garagash, Propagation of a plane-strain hydraulic fracture with a fluid lag : Early time solution, International Journal of Solids and Structures 43 (2006) 5811-5835.

[18] E. Papachristos, L. Scholts, F. Donz, B. Chareyre, Intensity and volumetric characterizations of hydraulically driven fractures by hydromechanical simulations. International Journal of Rock Mechanics and Mining Sciences 93 (2017) 163 - 178. doi: https://doi.org/10. 1016/j.ijrmms.2017.01.011 URL http://www.sciencedirect.com/science/article/pii/S136516091730076X

[19] P. Grassla, C. Fahy, D. Gallipoli, S. Wheeler, On a 2D hydro-mechanical lattice approach for modelling hydraulic fracture, Journal of the Mechanics and Physics of Solids 75 (2015) 104-118.

[20] P. Fu, S. Johnson, C. Carrigan, An explicitly coupled hydro-geomechanical model for simulating hydraulic fracturing in arbitrary discrete fracture networks, International Journal for Numerical and Analytical Methods in Geomechanics 37 (2013) 2278-2300.

[21] T. Belytschko, T. Black, Elastic crack growth in finite element with minimal remeshing, International Journal for Numerical Methods in Engineering 45 (1999) 601-620.

[22] N. Moës, J. Dolbow, T. Belytschko, A finite element method for crack growth without remeshing, International Journal for Numerical Methods in Engineering 46 (1999) 131-150.

[23] J.M.Melenk, I. Babuska, The partition of unity finite element method : Basic theory and applications, Comput. Methods Appl. Mech. Engrg. 139 (1996) 289-314.

[24] G. Barenblatt, The mathematical theory of equilibrium cracks in brittle fracture, Adv. Appl. Mech. 7 (1962) 55-130.

[25] R. Bargellini, J. Besson, E. Lorentz, S. Michel-Ponnelle, A non-local finite element based on volumetric strain gradient : application to ductile fracture, Computational Materials Science 33 (2009) 762-767.

[26] V. Hakim, A. Karma, Laws of crack motion and phase-field models of fracture, Journal of Mechanics and Physics of Solids 57 (2009) 342-368.

[27] M. Wheeler, T. Wick, W. Wollner, An augmented-lagrangian method for the phase field approach for pressurized fractures, Comput. Meth. Appl. Mech. Engng. 271 (2014) 69-85.

[28] C. Miehe, F. Welschinger, M. Hofacker, Thermodynamically consistent phase field models of fracture : Variational principles and multi-field fe implementations, Int. J. Numer. Meth. Engng. 83 (2010) 1273-1311.

[29] C. Comi, U. Perego, Numerical aspects of non-local damage analyses, Rev. Eur. Elem. Finis 10 (2001) 227-242

[30] G. Pijaudier-Cabot, Z. P. Bazant, Non local damage theory, J. Engng. Mech. 113 (1987) 1512-1533.

[31] B. Lecampion, An extended finite element method for hydraulic fracture problems, Communications in Numerical Methods in Engineering 25 (2009) 121-133

[32] E. Gordeliy, A. Peirce, Enrichment strategies and convergence properties of the XFEM for hydraulic fracture problems, Comput. Meth. Appl. Mech. Engng. 283 (2015) 474-502.

[33] E. Detournay, Propagation regimes of fluid-driven fractures in impermeable rocks, Int. J. Geomech 4 (2004) 1-11.

[34] M. Faivre, B. Paul, F. Golfier, R. Giot, P. Massin, D. Colombo, 2D coupled HM-XFEM modeling with cohesive zone model and applications to fluid-driven fracture network., Engineering Fracture Mechanics 59 (2016) 115-143.

[35] A.R.Khoei, S. Moallemi, E. Haghighat, Thermo-hydro-mechanical modelling of impermeable discontinuity in saturated porous media with X-FEM technique, Engineering Fracture Mechanics 96 (2012) 701-723.

[36] E. Gordeliy, A. Peirce, Coupling schemes for modeling hydraulic fracture propagation using the XFEM, Comput. Methods Appl. Mech. Engrg. 253 (2013) 305-322.

[37] E. Gordeliy, A. Peirce, Implicit level set schemes for modeling hydraulic fractures using the XFEM, Comput. Methods Appl. Mech. Engrg. 266 (2013) 125-143.

[38] T. Mohammadnejad, A.R.Khoei, An extended finite element method for fluid flow in partially saturated porous media with weak discontinuities; the convergence analysis of local enrichment strategies, Comput. Mech. 51 (2013) 327-345. 
[39] T. Mohammadnejad, A.R.Khoei, Hydro-mechanical modeling of cohesive crack propagation in multiphase porous media using the extended finite element method, International Journal for Numerical and Analytical Methods in Geomechanics 37 (2013) $1247-1279$.

[40] L. Simoni, S. Secchi, Cohesive fracture mechanics for a multi-phase porous medium, Engng. Comput. 20 (2003) $675-698$.

[41] A. Younes, P. Núñez, A. Makradi, Q. Shao, L. Bouhala, S. Belouettar, An xfem model for cracked porous media : effects of fluid flow and heat transfer, Int. J. Fracture 185 (2014) 155-169.

[42] A.R.Khoei, T. Mohammadnejad, Numerical modeling of multiphase fluid flow in deforming porous media : A comparison between two- and three-phase models for seismic analysis of earth and rockfall dams, Computers and Geotechnics 38 (2011) $142-166$.

[43] Q. Ren, Y. Dong, T. Yu, Numerical modelling of concrete hydraulic fracturing with extended finite element method, Sci. China Ser. E-Tech. Sci. 52 (2009) 559-565.

[44] J. Huygue, E. Remij, J. Remmers, D. Smeulders, The enhanced local pressure model for the accurate analysis of fluid pressure driven fractures in porous materials, Computer Methods in Applied Mechanics and Engineering 286 (2014) 293-312.

[45] A. Bunger, A. Peirce, Numerical simulation of simultaneous growth of multiple interacting hydraulic fractures from horizontal wells, ASCE Shale Energy Engineering Conference, 2014.

[46] A. Khoei, M. Vahab, M. Hirmand, Modeling the interaction between fluid-driven fracture and natural fault using an enriched-FEM technique, Int J Fract 197 (2016) 1-24.

[47] F. Shi, X. Wang, C. Liu, H. Liu, H. Wu, An XFEM-based method with reduction technique for modeling hydraulic fracture propagation in formations containing frictional natural fractures Engineering Fracture Mechanics 173 (2017) 64 - 90. doi : https://doi .org/10.1016/ j.engfracmech.2017.01.025

URL http://www.sciencedirect.com/science/article/pii/S0013794416305938

[48] H. Wang, Numerical modeling of non-planar hydraulic fracture propagation in brittle and ductile rocks using XFEM with cohesive zone method, Journal of Petroleum Science and Engineering 135 (2015) 127-140.

[49] H. Wang, Numerical investigation of fracture spacing and sequencing effects on multiple hydraulic fracture interference and coalescence in brittle and ductile reservoir rocks, Engineering Fracture Mechanics 157 (2016) 107-124.

[50] P. Gupta, C. Duarte, Simulation of non-planar three-dimensional hydraulic fracture propagation, Numerical and Analytical Methods in Geomechanics 38 (2014) 1397-1430.

[51] M. Haddad, K. Sepehrnoori, XFEM-based CZM for the simulation of 3D multiple-cluster hydraulic fracturing in quasi-brittle shale formations, Rock Mechanics and Rock Engineering 49 (2016) 4731-4748.

[52] E. Carter, Optimum fluid characteristics for fracture extension, Drill Prod. Pract. (1957) 261-270.

[53] J. Rethore, R. de Borst, M.A.Abellan, A two-scale approach for fluid flow in fractured porous media, International Journal For Numerical Methods In Engineering 71 (2007) 780-800.

[54] J. Rethore, R. de Borst, M.A.Abellan, A two-scale model for fluid flow in an unsaturated porous medium with cohesive cracks, Comput. Mech. 48 (2008) 227-238.

[55] S. Secchi, B. Schrefler, Hydraulic fracturing and its peculiarities, Asia Pacific Journal on Computational Engineering (1 :8 doi :10.1186/21961166-1-8).

[56] M. Ndeffo, P. Massin, N. Moës, A. Martin, S. Gopalakrishnan, On the construction of approximation space to model discontinuities and cracks with linear and quadratic extended finite elements, Adv. Model. and Simul. in Eng. Sciences 4 (2017) $6: 1-6: 51$.

[57] B. Paul, M. N'Deffo, P. Massin, N. Moës, An integration technique for 3D curved cracks and branched discontinuities within the extended finite element method, Finite Element in Analysis and Design 123 (2017) 19-50.

[58] G. Ferté, P. Massin, N. Moës, 3D crack propagation with cohesive elements in the extended finite element method, Computational Methods in Applied Mechanics and Engineering 300 (2016) 347-374.

[59] M. Siavelis, M. L. E. Guiton, P. Massin, N. Moës, Large sliding contact along branched discontinuities with X-FEM, Computational Mechanics 52 (2013) 201-219.

[60] R.W.Lewis, B.A.Schrefler, The finite element method in the static and dynamic deformation and consolidation of porous media 2 nd edition, Wiley Chichester, 1998.

[61] E. Lorentz, A mixed interface finite element for cohesive zone models, Comput. Methods Appl. Mech. Engrg. 198 (2008) $302-317$.

[62] S. Osher, J.A.Sethian, Fronts propagating with curvature dependent speed : algorithms based on Hamilton-Jacobi formulations, Journal of Computational Physics 79 (1988) 12-49.

[63] J. A. Sethian, Level set methods and fast marching methods (2nd edition).

[64] A. Hansbo, P. Hansbo, A finite element method for the simulation of strong and weak discontinuities in solid mechanics, Comput. Meth. Appl. Mech. Engrg. 193 (2004) 3523-3540.

[65] N. Moës, E. Bechet, M. Tourbier, Imposing dirichlet boundary conditions in the extended finite element method, International Journal For Numerical Methods in Engineering 67 (2006) 1641-1669.

[66] E. Bechet, N. Moës, B. Wohlmuth, A stable lagrange multiplier space for stiff interface conditions within the extended finite element method, International Journal For Numerical Methods in Engineering 78 (2009) 931-954.

[67] S. Geniaut, P. Massin, N. Moës, A stable 3D contact formulation for cracks using XFEM, Revue Europénne de Mécanique Numérique 75 (2007) 259-276.

[68] A. Bunger, E. Detournay, D. Garagash, Toughness-dominated hydraulic fracture with leak-off, Int. J. Fract. 134 (2005) 175-190.

[69] J. Adachi, E. Siebrits, A. Pierce, J. Desroches, Computer simulation of hydraulic fractures, International Journal of Rock Mechanics \& Mining Sciences 44 (2007) 739-757.

[70] Y. Kovalyshen, Fluid-driven fracture in poroelastic medium, Ph.D. thesis, The University of Minnesota (2010).

[71] A. Gravouil, N. Moës, T. Belytschko, Non-planar 3D crack growth by the extended finite element and level sets - Part II : Level set update, Int. J. Numer. Meth. Engng. 53 (2002) 2569-2586.

[72] M. Duflot, A study of the representation of cracks with the level sets, Int. J. Numer. Meth. Engng. 70 (2007) $1261-1302$.

[73] F. Erdogan, G. Sih, On the crack extension in plane loading and transverse shear, Journal Basic Engng. 85 (1963) 519-527.

[74] M. Haddad, K. Sepehrnoori, Simulation of hydraulic fracturing in quasi-brittle shale formations using characterized cohesive layer : Stimu- 
lation controlling factors, Journal of Unconventional Oil and Gas Resources 9 (2014) 65-83.

[75] C. Daux, N. Moës, J. Dolbow, N. Sukumar, T. Belytschko, Arbitrary branched and intersecting cracks with the extended finite element method, Int. J. Numer. Meth. Engng. 48 (2000) 1741-1760.

[76] A. Simone, C. Duarte, E. V. der Giessen, A generalized finite element method for polycristals with discontinous grain boundaries, Int. J. Numer. Meth. Engng. 67 (2006) 1122-1145.

[77] T. Strouboulis, K. Copps, I. Babuska, The generalized finite element method : an example of its implementation and illustration of its performance, International Journal for Numerical Methods in Engineering 47 (2000) 1401-1417.

[78] A. Duarte, L. Reno, A. Simone, A high-order generalized FEM for through-the-thickness branched cracks, International Journal for Numerical Methods in Engineering 72 (2007) 325-351.

[79] J. Sanders, J. Dolbow, T. Laursen, On methods for stabilizing constraints over enriched interfaces in elasticity, Int. J. Numer. Meth. Engng. 78 (2009) 1000-1036.

[80] M. Siavelis, Modélisation numérique X-FEM de grands glissements avec frottement le long d'un réseau de discontinuités, Ph.D. thesis, Ecole Centrale de Nantes (2011).

[81] A. Pouya, Three-dimensional flow in fractured porous media : a potential solution based on singular integral equations, Advances in Water Ressources 35 (2012) 30-40.

[82] M. Vu, A. Pouya, D. Seyedi, Modelling of steady-state fluid flow in 3D fractured isotropic porous media : applications to effective permeability calculation, Numerical and Analytical Methods in Geomechanics 37 (2012) 2257-2277.

[83] M.K.Fisher, J.R.Heinze, C.D.Harris, B.M.Davidson, C.A.Wright, K.P.Dunn, Optimizing horizontal completion techniques in the Barnett Shale using microseismic fracture mapping, in : Paper SPE 90051 presented at the 2004 SPE Annual Technical Conference and Exhibition, Houston TX, September 26-29.

[84] Y. Cheng, Boundary element analysis of the stress distribution around multiple fractures : Implications for the spacing of perforation clusters of hydraulically fractured horizontal wells, in : SPE 125769, 2009.

[85] A.P.Bunger, Analysis of the power input needed to propagate multiple hydraulic fractures, International Journal of Solids and Structures 50 (2013) 1538-1549.

[86] B. Lecampion, J. Desroches, Simultaneous initiation and growth of multiple radial hydraulic fractures from horizontal wellbore, Journal of the Mechanics of Physics and Solids 82 (2015) 235-258.

[87] N. Moës, C. Stolz, P. E. Bernard, N. Chevaugeon, A level set based model for damage growth : the thick level set approach, Int. J. Numer. Meth. Engng. 86 (2011) 358-380. 